\title{
The Possible Role of TASK Channels in Rank-Ordered Recruitment of Motoneurons in the Dorsolateral Part of the Trigeminal Motor Nucleus
}

\author{
Keiko Okamoto, ${ }^{1,2 *}$ Norihito Emura, ${ }^{1,2 *}$ Hajime Sato, ${ }^{1 *}$ Yuki Fukatsu, ${ }^{1,3 *}$ @Mitsuru Saito, ${ }^{1,7 *}$ Chie
} Tanaka, ${ }^{1,3}$ Yukako Morita, ${ }^{1,2}$ Kayo Nishimura, ${ }^{1,2}$ Eriko Kuramoto, ${ }^{4,8}$ Dong Xu Yin, ${ }^{1}$ (1) Kazuharu Furutani, ${ }^{5}$ (1) Makoto Okazawa, ${ }^{6}$ Yoshihisa Kurachi, ${ }^{5}$ Takeshi Kaneko, ${ }^{4}$ Yoshinobu Maeda, ${ }^{3}$ Takashi Yamashiro, ${ }^{2}$ (1) Kenji Takada, ${ }^{2}$ (D) Hiroki Toyoda, ${ }^{1}$ and Youngnam Kang ${ }^{1}$

DOI:http://dx.doi.org/10.1523/ENEURO.0138-16.2016

${ }^{1}$ Department of Neuroscience and Oral Physiology, Osaka University Graduate School of Dentistry, Suita, Osaka 5650871, Japan, ${ }^{2}$ Department of Orthodontics and Dentofacial Orthopedics, Osaka University Graduate School of Dentistry, Suita, Osaka 565-0871, Japan, ${ }^{3}$ Department of Removable Prosthodontics, Osaka University Graduate School of Dentistry, Suita, Osaka 565-0871, Japan, ${ }^{4}$ Department of Morphological Brain Science, Graduate School of Medicine, Kyoto University, Kyoto 606-8501, Japan, ${ }^{5}$ Department of Pharmacology, Graduate School of Medicine, Osaka University, Suita, Osaka 565-0871, Japan, ${ }^{6}$ Department of Vascular Physiology, National Cardiovascular Center Research Institute, Suita, Osaka 565-8565, Japan, ${ }^{7}$ Department of Oral Physiology, Graduate School of Medical and Dental Sciences, Kagoshima University, Kagoshima 890-8544, Japan, ${ }^{8}$ Department of Oral Anatomy and Cell Biology, Graduate School of Medical and Dental Sciences, Kagoshima University, Kagoshima 890-8544, Japan

\begin{abstract}
Because a rank-ordered recruitment of motor units occurs during isometric contraction of jaw-closing muscles, jaw-closing motoneurons (MNs) may be recruited in a manner dependent on their soma sizes or input resistances (IRs). In the dorsolateral part of the trigeminal motor nucleus (dl-TMN) in rats, MNs abundantly express TWIK (two-pore domain weak inwardly rectifying $\mathrm{K}$ channel)-related acid-sensitive- $\mathrm{K}^{+}$channel (TASK)-1 and TASK3 channels, which determine the IR and resting membrane potential. Here we examined how TASK channels are involved in IR-dependent activation/recruitment of MNs in the rat dl-TMN by using multiple methods. The real-time PCR study revealed that single large MNs $(>35 \mu \mathrm{m})$ expressed TASK1 and TASK3 mRNAs more abundantly compared with single small MNs (15-20 $\mu \mathrm{m})$. The immunohistochemistry revealed that TASK1 and TASK3 channels were complementarily distributed in somata and dendrites of MNs, respectively. The density of TASK1 channels seemed to increase with a decrease in soma diameter while there were inverse relationships between the soma size of MNs and IR, resting membrane potential, or spike threshold. Dual whole-cell recordings obtained from smaller and larger MNs revealed that the recruitment of MNs depends on their IRs in response to repetitive stimulation of the presumed la afferents. 8-Bromoguanosine-cGMP decreased IRs in small MNs, while it hardly changed those in large MNs, and subsequently decreased the difference in spike-onset latency between the smaller and larger MNs, causing a synchronous activation of MNs. These results suggest that TASK channels play critical roles in rank-ordered recruitment of MNs in the dl-TMN.
\end{abstract}

\section{Significance Statement}

The mastication of foods occurs during the slow-closing phase of the mastication cycle, in which the isometric contraction of jaw-closing muscles is developed through the rank-ordered recruitment of jaw-closing motoneurons (MNs). However, its molecular mechanism remains unknown. Here we show that the TWIK (two-pore domain weak inwardly rectifying $\mathrm{K}$ channel)-related acid-sensitive- $\mathrm{K}^{+}$channel (TASK)-1 and TASK3 channels, which determine the input resistance and resting membrane potential, are differentially expressed between small and large MNs, and play critical roles in the rank-ordered recruitment of jaw-closing MNs. The principle of the rank-ordered recruitment was originally proposed based on the conduction velocity of the motor nerve fibers innervating a set of muscle fibers. After a half-century, we for the first time disclosed the molecular mechanism for the rank-ordered recruitment of jaw-closing MNs. 
Key words: input resistance; leak potassium channel; recruitment; TASK channel; trigeminal motoneuron

\section{Introduction}

TWIK (two-pore domain weak inwardly rectifying $\mathrm{K}$ channel)-related acid-sensitive- $\mathrm{K}^{+}$channel (TASK)-1 and TASK3 channels are the major determinants of the resting membrane potential and input resistance (IR; Lauritzen et al., 2003; Enyedi and Czirják, 2010). TASK1 and TASK3 channel subunits can assemble into dimers; TASK1 homodimeric, TASK3 homodimeric, and TASK $1 / 3$ heterodimeric channels (Berg et al., 2004; Enyedi and Czirják, 2010). These channels show different $\mathrm{pH}$ sensitivities (Czirják and Enyedi, 2002). The single-channel conductance of TASK3 homodimeric or TASK $1 / 3$ heterodimeric channels is two times larger compared with TASK1 homodimeric channels (Kang et al., 2004).

Motoneurons (MNs) in the dorsolateral part of the trigeminal motor nucleus (dl-TMN; Mizuno et al., 1975; Limwongse and DeSantis, 1977) highly express TASK1 and TASK3 mRNAs (Karschin et al., 2001; Talley et al., 2001). During voluntary isometric contraction, a rank-ordered recruitment of motor units occurs, which is well known as the size principle (Henneman et al., 1965; Henneman, 1991). A small motor unit is composed of a small $M N$ and a small number of the thin muscle fibers that it innervates, while a large motor unit is composed of a large $\mathrm{MN}$ and a large number of the thick muscle fibers it innervates (Burke, 1975). Such a rank-ordered recruitment of motor units is mediated by the orderly recruitment of MNs, depending on the order of their axonal conduction velocities (Bawa et al., 1984). The axonal conduction velocity of MNs is proportional to their soma sizes, and it is generally believed that the IR in MNs is inversely proportional to their size. Then, it can be assumed that the smaller MNs with larger IRs are more easily activated than the larger MNs with smaller IRs in response to the same synaptic inputs, which is known as the Henneman size principle. However, it was proposed that the specific membrane resistance, but not the cell size itself, is the key determinant of the orderly recruitment of spinal $\alpha \mathrm{MNs}$, although the actual sizes of MNs were not measured (Gustafsson and Pinter, 1984a,b). Despite some controversy about the role of size in the orderly recruitment of MNs, the relation-

Received May 12, 2016; accepted June 21, 2016; First published July 7, 2016. The authors declare no competing financial interests.

Author contributions: Y. Kang designed research. K.O., N.E., H.S., Y.F., M.S., C.T., Y.M., K.N., E.K., D.X.Y., K.F. M.O. and H.T. performed research. K.O., N.E., H.S., Y.F., M.S., C.T., Y.M., K.N., E.K., D.X.Y., K.F., M.O., Y. Kurachi, T.K., Y.M., T.Y., K.T., H.T., and Y. Kang analyzed the data. Y. Kurachi and H.T. wrote the paper.

This study was supported by Grant-in-Aid for Scientific Research (B) 26290006; to Y. Kang).

*K.O., N.E., H.S., Y.F., and M.S. contributed equally to this work.

Correspondence should be addressed to either Dr. Youngnam Kang or Dr. Hiroki Toyoda, Department of Neuroscience and Oral Physiology, Osaka University Graduate School of Dentistry, 1-8, Yamadaoka, Suita, Osaka 565-0871, Japan, E-mail: kang@dent.osaka-u.ac.jp or toyoda@dent.osaka-u.ac.jp.

DOI:http://dx.doi.org/10.1523/ENEURO.0138-16.2016

Copyright (C) 2016 Okamoto et al.

This is an open-access article distributed under the terms of the Creative Commons Attribution 4.0 International, which permits unrestricted use, distribution and reproduction in any medium provided that the original work is properly attributed. ships between the sizes of MNs and IRs, between the sizes of MNs and resting membrane potentials, and between the sizes of MNs and spike thresholds have not been investigated in relation with TASK channels. Because the orderly recruitment of motor units is seen during the voluntary isometric contraction of human jawclosing (JC) muscles (Yemm, 1977), the MNs located in the dl-TMN (Mizuno et al., 1975; Limwongse and DeSantis, 1977) and innervating JC muscles are likely to be recruited, depending on their sizes or IRs. Then, the expression levels and/or patterns of TASK channels would differ, depending on the sizes of MNs in the dl-TMN.

TASK1 channels are inhibited by various neurotransmitters through the activation of G-protein-coupled receptors (Bayliss et al., 2003; Lesage, 2003). On the other hand, it has been previously reported that TASK1-like leak $\mathrm{K}^{+}$currents in basal forebrain cholinergic neurons are activated by nitric oxide (NO) donor and 8-bromoguanosine-cGMP (8-Br-cGMP; Kang et al., 2007b; Toyoda et al., 2008). It was also found that 8-Br-cGMP enhances TASK1 currents in TASK1-expressed HEK cells, and this effect is brought about by the activation of cGMP-dependent protein kinase (PKG; Toyoda et al., 2010). Nitrergic neurons innervate trigeminal MNs (Abudara et al., 2002), and neuronal NO synthesis is expressed in $\sim 10 \%$ of the premotor neurons projecting to the TMN (Pose et al., 2005). Although the percentage of premotor neurons that express neuronal NO synthesis seems to be low, the numerous nitrergic premotor fibers are in close apposition to trigeminal MN processes (Abudara et al., 2002), and NO is a diffusible gas that readily permeates cell membranes (Bredt et al., 1991). Thus, NO would play an important role in modulating the activity of MNs in the dl-TMN. Provided that the TASK channels are responsible for the rank-ordered recruitment of MNs, it is possible that $\mathrm{NO}$ modulates the rank-ordered recruitment of $\mathrm{MNs}$ in the dl-TMN.

In the present study, we first investigated whether and how expression levels and/or patterns of TASK channels are different between small and large MNs in the dl-TMN using quantitative real-time PCR and immunohistochemistry. We then examined how MNs in the dl-TMN are recruited by repetitive stimulation of the presumed la afferents, and the effects of $8-\mathrm{Br}$-cGMP on the recruitment of MNs using dual whole-cell current-clamp recordings and voltage-sensitive dye imaging.

\section{Materials and Methods}

All experiments were performed in accordance with the approvals of the animal ethics committees of our institutions for the care and use of laboratory animals.

\section{Laser capture microdissection and real-time RT-PCR}

Wistar rats of both sexes at postnatal days 15-17 (Nihon Dobutsu) were anesthetized with chloral hydrate (400 $\mathrm{mg} / \mathrm{kg}$, i.p.) and transcardially perfused with PBS. The brain was quickly removed from the skull and immediately flash frozen with dry ice. Serial cryostat sections $(20 \mu \mathrm{m})$ of the dl-TMN were cut and mounted on polyethylene 
$\mathbf{A}_{\mathrm{a}}$

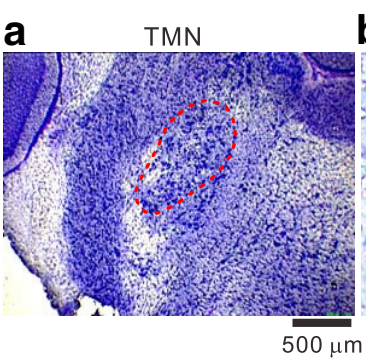

b Small MN

C Large MN

d Laser Microdissection
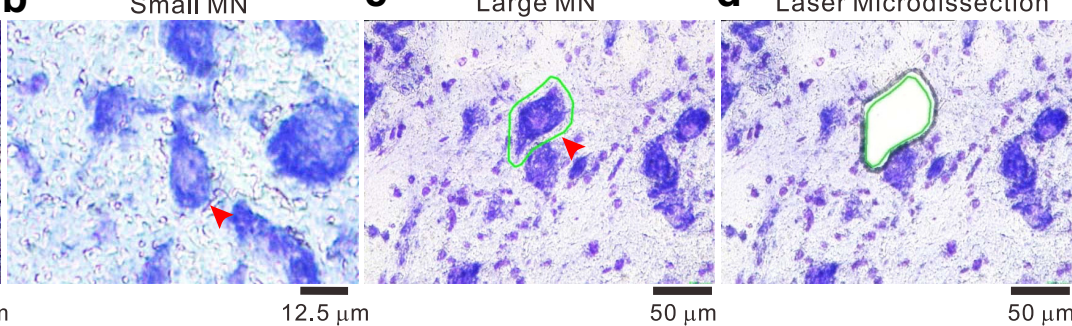

B

$\mathrm{C}_{\mathrm{a}}$

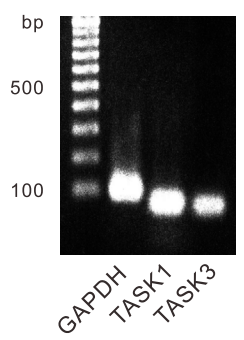

D

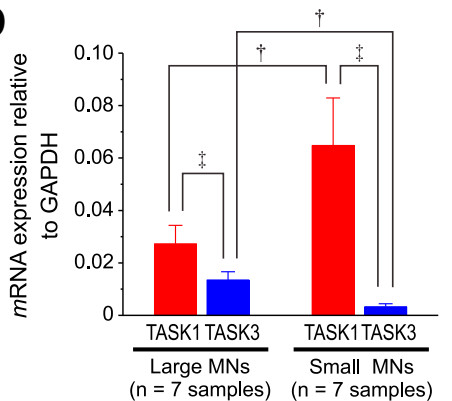

Large MNs

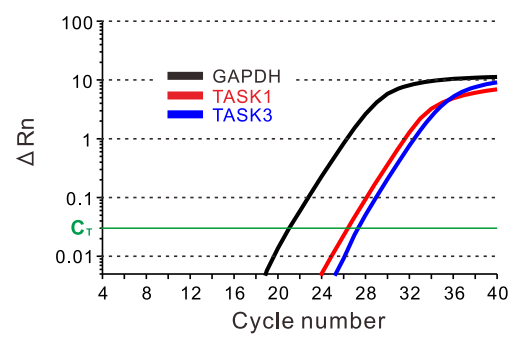

E

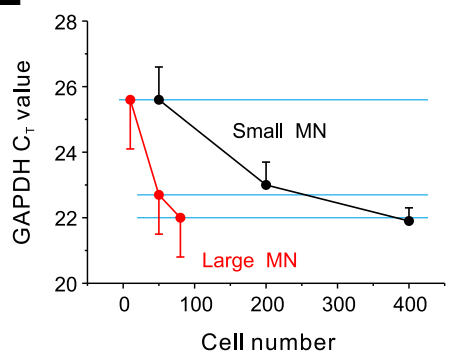

b

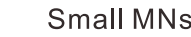

$F$

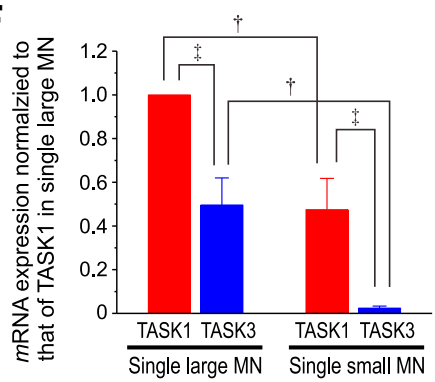

Figure 1. TASK1 and TASK3 mRNA expressions in small and large MNs in the dl-TMN. Aa, Toluidine blue staining of the brainstem slice including the dl-TMN. $\boldsymbol{A} \boldsymbol{b}, \boldsymbol{A} \boldsymbol{c}$, Arrowheads showing small MNs $(\boldsymbol{A} \boldsymbol{b})$ and large MNs $(\boldsymbol{A c})$. A margin of the large MN depicted with a green line $(\boldsymbol{A c})$. $\boldsymbol{A d}$, An image of the same section as in $\boldsymbol{A c}$ after laser microdissection. $\boldsymbol{B}$, TASK1 and TASK3 mRNA expression in large MNs revealed by standard RT-PCR. $\mathbf{C a}, \mathbf{C b}$, Quantitative real-time PCR analysis of TASK1 and TASK3 mRNA expression compared with GAPDH mRNA in large MNs $(\mathbf{C a})$ and small MNs $(\mathbf{C b})$. The $\triangle \mathrm{Rn}$ was plotted against the number of cycles. The mRNA copy number of TASK3 was slightly higher than that of TASK1 in larger MNs, while the former was apparently larger than the latter in small MNs. $\boldsymbol{D}$, The mean expression levels of TASK1 and TASK3 mRNAs relative to GAPDH mRNA in 80 large MNs and in 400 small MNs ( $n=7$ and $n=7$ samples, respectively). $\ddagger p<0.001$, paired $t$ test; $\nmid p<0.001$, unpaired $t$ test. $\boldsymbol{E}$, The relationships between cell numbers and $\mathrm{C}_{\mathrm{T}}$ values of GAPDH mRNA obtained from 10, 50, and 80 large MNs $(n=7, n=5$, and $n=5$ samples, respectively) and those obtained from 50, 200, and 400 small MNs $(n=6, n=6$, and $n=5$ samples, respectively). $\boldsymbol{F}$, The expression levels of TASK1 and TASK3 mRNAs in a single large MN and those in a single small MN. The respective mRNA expression levels were normalized to that of TASK1 mRNA in a single large MN. $\ddagger p<0.001$, paired $t$ test; $\dagger p<0.001$, unpaired $t$ test.

terephthalate (PET) membrane slides (Leica Microsystems). Sections were air dried at room temperature for 30 $\mathrm{s}$, fixed with $70 \%$ ethanol for $1 \mathrm{~min}$, washed in RNase-free diethylpyrocarbonate (DEPC)-treated water and stained with $0.05 \%$ toluidine blue for $1 \mathrm{~min}$ (Fig. 1 Aa). After rinsing twice in DEPC water for $1 \mathrm{~min}$, the sections were air dried for $5 \mathrm{~min}$ and immediately subjected to laser microdissection (LMD).

PET slides were mounted on a Leica AS LMD System (Leica Microsystems) with the section facing downward. Dye-labeled MNs were selected for capture and divided into two groups according to their mean diameters. The neuron whose mean diameter was 15-20 $\mu \mathrm{m}$ was categorized as a small MN (Fig. 1Ab), while the neuron whose mean diameter was $>35 \mu \mathrm{m}$ was categorized as a large
MN (Fig. 1Ac). After adjusting intensity, aperture, and cutting velocity, the pulsed UV laser beam was carefully directed along the border of the neuron (Fig. 1Ad). The cut area was then transferred by gravity alone into a microcentrifuge tube cap placed directly underneath the section. The tube cap was filled with a guanidine isothiocyanatecontaining buffer (Buffer RLT, Qiagen) to ensure the isolation of intact RNA. For each experiment, 80 large MNs were sampled from one to two brains, and 400 small MNs were sampled from two to three brains. The 80 large or 400 small MNs were collected in one microcentrifuge tube as one sample (see below).

Total RNA was extracted using the RNeasy Micro Kit (Qiagen) according to the manufacturer instructions, during which samples were subjected to DNase digestion 
using DNase I (Qiagen) to remove any contaminating genomic DNA. RNA was then reverse transcribed for $1 \mathrm{~h}$ at $37^{\circ} \mathrm{C}$ using Sensicript Reverse Transcription kit (Qiagen). Real-time PCR was performed in a reaction mixture $(20 \mu \mathrm{l})$ composed of $10 \mu \mathrm{l}$ of $2 \times$ Fast SYBR Green Master Mix (Applied Biosystems), $0.35 \mathrm{~mm}$ each primer and $2 \mu \mathrm{l}$ of reverse transcription product, through the following series of reactions: $95^{\circ} \mathrm{C}$ for $20 \mathrm{~s}$, followed by 40 cycles $\left(95^{\circ} \mathrm{C}, 3 \mathrm{~s} ; 60^{\circ} \mathrm{C}, 30 \mathrm{~s}\right)$, with a Step One Real-Time PCR System (Applied Biosystems). The following primers were used for PCR: rat TASK-1: forward, 5'-CGGCTTCCGCAA CGTCTAT-3', and reverse, 5'-TTGTACCAGAGGCACGAGCA-3'; rat TASK-3: forward, 5'-GACGTGCTGAG GAACACCTACTT-3', and reverse, 5'-GTGTGCATTCCA GGAGGGA-3'; and GAPDH: forward, 5'-GAGAATGGG AAGCTGGTCATCAAC-3' and reverse, 5'-ACTCCACGACA TACTCAGCACCAG-3'. Standard curves were generated for each set of primers using serial dilutions of rat brain cDNA to ensure a similar efficiency of amplification (TASK1, 100\%; TASK3, 100\%). All the primers were obtained from Sigma Genosys. Aliquots of the PCR products were analyzed by $2 \%$ agarose gel electrophoresis (Nacalai Tesque) and were visualized using ethidium bromide. The PCR product was run in parallel with known molecular weight markers (100 bp ladder, Bio-Rad). The amplified PCR fragments obtained from large MNs were detected in accordance with the respective lengths predicted by the primers (Fig. 1B). The data were analyzed using the comparative cycle-threshold $\left(\mathrm{C}_{\mathrm{T}}\right)$ method (Pfaffl, 2001), where the amount of target is normalized to an endogenous reference gene, GAPDH.

\section{Evaluation of size-dependent expression of GAPDH mRNA}

Because the housekeeping gene GAPDH mRNA level is likely to be proportional to the cell volume, the GAPDH mRNA levels obtained from a certain number of small MNs $\left(N_{S}\right)$ and a certain number of large MNs $\left(N_{L}\right)$ are proportional to $N_{S} \times V_{S}$ and $N_{L} \times V_{L}$, respectively, given that $V_{S}$ and $V_{L}$ are the mean cell volumes of the sampled small and large MNs, respectively. When $N_{S} \times V_{S}$ is equal to $N_{L} \times V_{L}, V_{S} / V_{L}$ is equal to $N_{L} / N_{S}$. The normalized GAPDH mRNA in a single small MN compared with that in a single large $M N$ is proportional to $N_{L} / N_{S}$. Therefore, we evaluated the relationship between the cell number and $\mathrm{C}_{\mathrm{T}}$ of GAPDH mRNA obtained from 50,200 , and 400 small MNs ( $n=6, n=6$, and $n=5$ samples, respectively) and that between the cell number and $\mathrm{C}_{\mathrm{T}}$ of GAPDH mRNA obtained from 10, 50, and 80 large MNs $(n=7, n$ $=5$, and $n=5$ samples, respectively). $\mathrm{C}_{\mathrm{T}}$ represents the fractional cycle number at which the baseline-corrected normalized fluorescence signal $(\Delta R n)$ reaches a threshold value (0.03)

\section{Anti-TASK1 antibody}

Peptide YKSREKLQYSIPMIIPRDLSTSDTC, which corresponds to C-terminal 25 aa (residues 332-356) of rat TASK1 (Leonoudakis et al., 1998) was synthesized (ThermoFisher Scientific). Cysteine in the C-terminal of the peptide was used for conjugation by a maleimide method. The peptide was conjugated with an equal weight of maleimide-activated bovine serum album (ThermoFisher Scientific). Four female guinea pigs (200 g; Shimizu Experimental Materials) were immunized by intracutaneous injections of the conjugate $(0.5 \mathrm{mg} / \mathrm{animal})$ in Freund's complete adjuvant (Difco), and, 4 weeks later, in incomplete adjuvant. The sera were recovered 14 days after the second immunization. The antibody was purified by ammonium sulfate fractionation ( $50 \%$ saturation), followed by affinity chromatography on a SulfoLink gel (ThermoFisher Scientific) coupled with peptide (2 $\mathrm{mg}$ peptide/ml gel). The antibody was eluted with $0.1 \mathrm{~m}$ glycine- $\mathrm{HCl}, \mathrm{pH}$ 2.5 , and $0.1 \mathrm{~m}$ tetraethyl ammonium, $\mathrm{pH} 11.8$, and was used for the following experiments.

The specificity of anti-TASK1 antibody was examined using HEK293 cells transfected with TASK1 or mock. For the transient expression of TASK1, HEK293 cells (DS Pharma Biomedical) cultured in DMEM supplemented with $10 \%$ FBS, $100 \mathrm{U} / \mathrm{ml}$ penicillin, and $100 \mu \mathrm{g} / \mathrm{ml}$ streptomycin were transfected with pIRES2-ZsGreen1-TASK1 by using Lipofectamine LTX (Invitrogen) or the empty vector pIRES2-ZsGreen1 (mock; Toyoda et al., 2010). For immunostaining of transient transfected cell lines, cells plated onto glass coverslips $4 \mathrm{~h}$ after transfection were fixed with $3 \%$ formaldehyde, $75 \%$ saturated picric acid, and $0.1 \mathrm{~m}$ phosphate- $\mathrm{NaOH}$ buffer at $\mathrm{pH}$ 7.2. The cells were stored at $4^{\circ} \mathrm{C}$ in PBS containing $0.02 \%$ sodium azide. The cells were incubated overnight with $1 \mu \mathrm{g} / \mathrm{ml}$ affinity-purified guinea pig anti-rat TASK1 antibody in PBS containing $0.3 \%$ Triton X-100, $0.12 \% \quad \lambda$-carrageenan, $0.02 \%$ sodium azide, and $1 \%$ normal donkey serum (PBS-XCD); and then were incubated for $1 \mathrm{~h}$ with $10 \mu \mathrm{g} / \mathrm{ml}$ biotinylated donkey anti-guinea pig IgG antibody (RRID: AB[lowen]2341097, Jackson ImmunoResearch) in PBS$X C D$. Subsequently, the cells were incubated for $1 \mathrm{~h}$ with avidin-biotin enzyme complex (ABC; VECTASTAIN Elite $A B C$, Vector Laboratories) in PBS containing $0.3 \%$ Triton $X-100$ (PBS-X). Furthermore, the sections were incubated for 30 min with biotinylated tyramine (BT)-glucose oxidase (GO) reaction mixture containing $1.25 \mu \mathrm{M} \mathrm{BT}, 3 \mu \mathrm{g} / \mathrm{ml}$ GO (259 U/mg; Nacalai Tesque), $2 \mathrm{mg} / \mathrm{ml} \beta$-D-glucose, and $1 \% \mathrm{BSA}$ in $0.1 \mathrm{M} \mathrm{PB}, \mathrm{pH}$ 7.4. After a rinse with PBS-X, the sections were incubated for $1 \mathrm{~h}$ with $5 \mu \mathrm{g} / \mathrm{ml}$ Alexa Fluor 594-conjugated streptavidin (RRID: AB[lowen]2313574; Invitrogen) to stain TASK1 and then were incubated for 5 min with DAPI to stain nuclei.

\section{Fluorescence immunohistochemistry}

For immunostaining, fixed brains were prepared from three Wistar rats following the protocol described in the previous study (Toyoda et al., 2010). The $40-\mu \mathrm{m}$-thick coronal sections of the fixed brains were incubated overnight in PBS-XCD with $2 \mu \mathrm{g} / \mathrm{ml}$ anti-rat TASK1 guinea pig antibody or $1 \mu \mathrm{g} / \mathrm{ml}$ anti-rat TASK3 (residues 57-73) rabbit antibody (RRID: AB[lowen]2039953, Alomone Labs) with or without anti-ChAT goat antiserum [AB144P (RRID: $A B$ [lowen]2079751), Chemicon] diluted 1:500. After a wash with PBS, the sections were incubated for $2 \mathrm{~h}$ with $10 \mu \mathrm{g} / \mathrm{ml}$ biotinylated anti-guinea pig IgG donkey antibody (Jackson ImmunoResearch) or $10 \mu \mathrm{g} / \mathrm{ml}$ biotinylated anti-rabbit IgG donkey antibody (RRID: AB[lowen]2340593; Jackson Immu- 

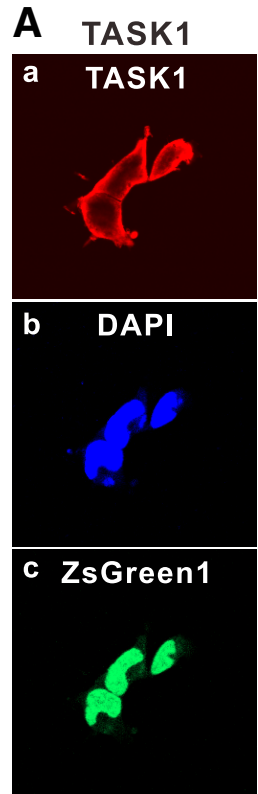

d Merged

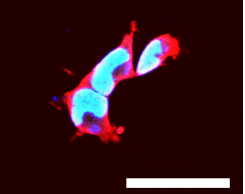

B Mock
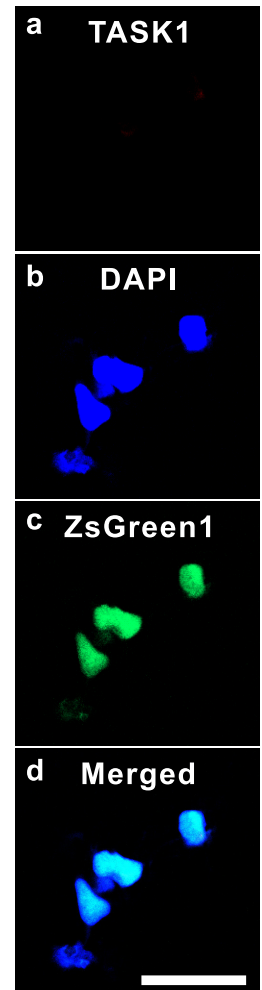

C ChAT

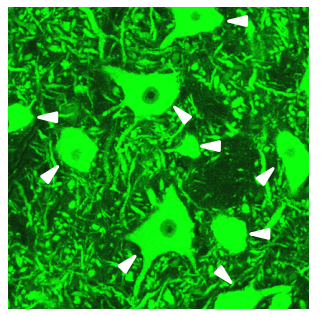

TASK1

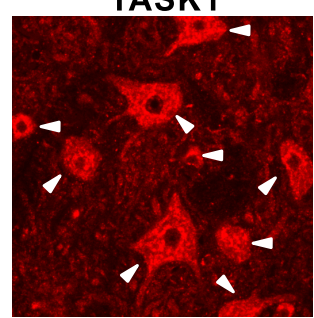

Merged

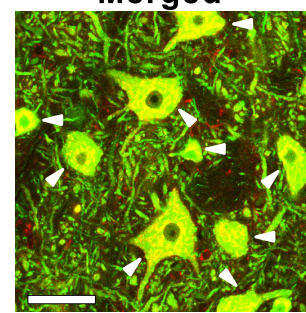

D ChAT

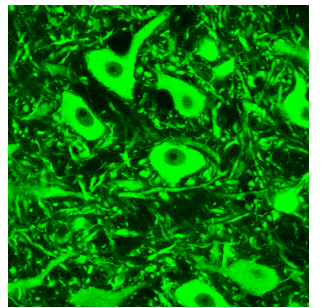

TASK1 absorption

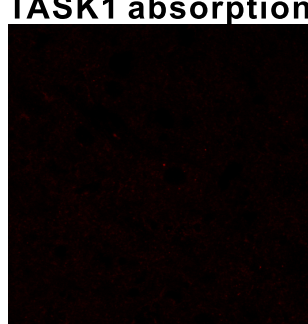

Merged

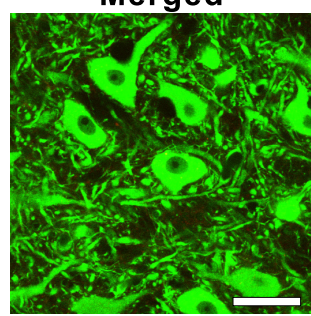

E ChAT

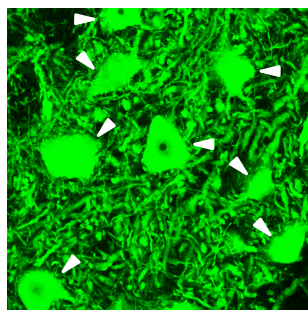

TASK3

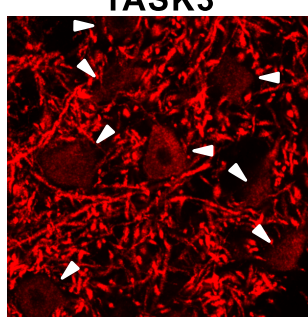

Merged

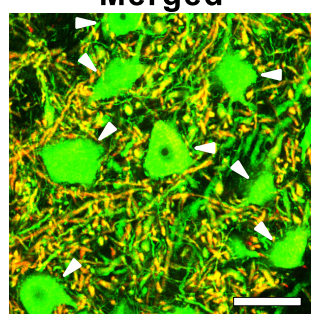

Figure 2. Complementary distribution of TASK1 and TASK3 channels in MNs in the dl-TMN. Aa-Ad, HEK cells transfected with TASK1 and ZsGreen1 displaying immunoreactivity along the plasma membrane and partly in the cytoplasm (Aa); nuclei staining with DAPI $(\boldsymbol{A} \boldsymbol{b})$; TASK1 transfection ensured with ZsGreen1 $(\boldsymbol{A c})$; and a merged fluorescence image $(\boldsymbol{A d})$. $\boldsymbol{B a}-\boldsymbol{B d}$, HEK cells transfected with mock and ZsGreen1 displaying no immunoreactivity $(\mathbf{B a})$; nuclei staining with DAPI $(\boldsymbol{B b})$; mock transfection ensured with ZsGreen1 (Bc); and a merged fluorescence image (Bd). C , Confocal photomicrographs showing immunoreactivity for ChAT (green) and TASK1 (red) in MNs. As revealed in the merged image, the TASK1 immunoreactivity was seen in somata (filled arrowheads) but not in dendrites. $\boldsymbol{D}$, Confocal photomicrographs showing the elimination of TASK1 immunoreactivity in ChAT (green)-positive MNs following the absorption of anti-TASK1 antibody by preincubating with the antigen peptide of TASK1. E, Confocal photomicrographs showing immunoreactivity for ChAT (green) and TASK3 (red) in MNs. As revealed in the merged image, the TASK3 immunoreactivity was primarily seen in dendrites but not in somata (filled arrowheads). Scale bars: $\boldsymbol{A}-\boldsymbol{E}, 50 \mu \mathrm{m}$.

noResearch) and then incubated for $1 \mathrm{~h}$ with $\mathrm{ABC}$ in PBS-X. For the immunostaining of TASK1, the sections were then incubated for 30 min with BT-GO reaction mixture, followed by incubation for $1 \mathrm{~h}$ with $5 \mu \mathrm{g} / \mathrm{m}$ Alexa Fluor 594conjugated streptavidin. For the immunostaining of TASK3, the sections were then incubated for $30 \mathrm{~min}$ with TSA Cyanine 3 (Су3) System (PerkinElmer). For the immunostainings of ChAT, the sections were then incubated for 2 h with $10 \mu \mathrm{g} / \mathrm{ml}$ Alexa Fluor 488-conjugated anti-goat IgG donkey antibody (RRID: AB[lowen]2534102; Invitrogen) and Cy3-conjugated streptavidin (Invitrogen) in the presence of $10 \%$ normal rabbit serum. For control experiments, when one of the primary antibodies was omitted or replaced with normal IgG or serum, no immunofluorescence for the omitted or replaced antibody was detected. For the absorption tests for anti-TASK1 and anti-TASK3 antibodies, the primary antibody solution was preincubated for $1 \mathrm{~h}$ with the antigen peptide (1 $\mu \mathrm{g}$ peptide for 1 $\mu \mathrm{g}$ antibody) prior to the incubation with sections (Fig. 2D, TASK1 absorption test). The sections were observed with a confocal laser-scanning microscope (LSM510, Zeiss).
Alexa Fluor 488 and 594 were excited with 488 and 543 $\mathrm{nm}$ laser beams, and observed through emission filters of 505-530 and >560 nm, respectively. The digital images were captured using LSM510 software (Zeiss).

\section{Slice preparation}

The procedure for slice preparation was the same as that in the previous study (Kang et al., 2007a). Using Wistar rats of both sexes at postnatal days 7-14 (Nihon Dobutsu), coronal sections with $250 \mu \mathrm{m}$ thickness, including the dl-TMN, were cut.

\section{Whole-cell recording from MNs in the dl-TMN}

The electrophysiological studies were performed on the MNs in the dl-TMN. Axopatch 200B (MDS Analytical Technologies; Figs. 3-6) and Axoclamp 2B (MDS; Fig. 7) were used for whole-cell patch-clamp recordings. The standard extracellular solution had the following composition (in mM): $124 \mathrm{NaCl}, 1.8 \mathrm{KCl}, 2.5 \mathrm{CaCl}_{2}, 1.3 \mathrm{MgCl}_{2}, 26$ $\mathrm{NaHCO}_{3}, 1.2 \mathrm{KH}_{2} \mathrm{PO}_{4}$, and 10 glucose, bubbled with a mixture of $95 \% \mathrm{O}_{2} / 5 \% \mathrm{CO}_{2}$. The internal solution had the 
A

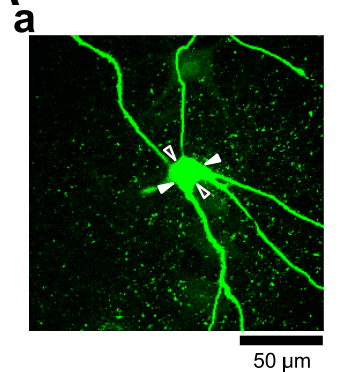

b

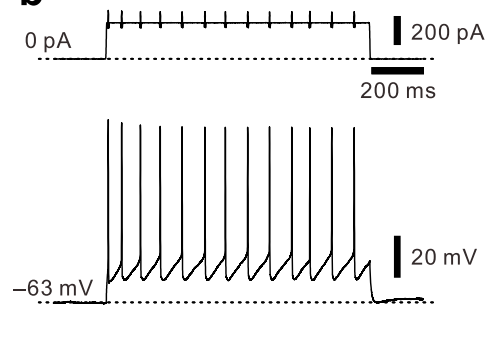

B

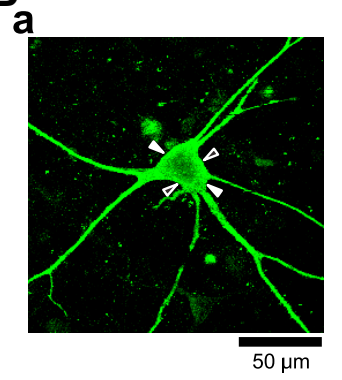

b

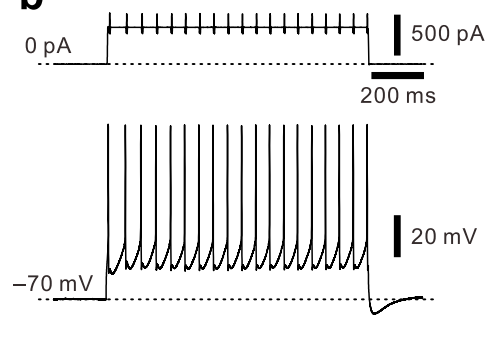

C

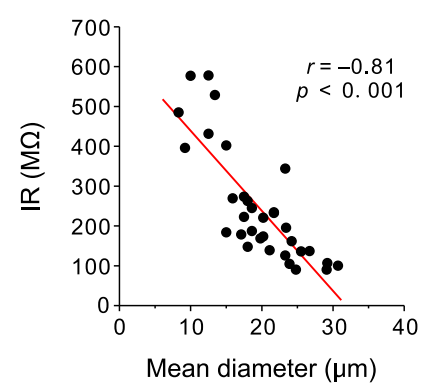

D

E

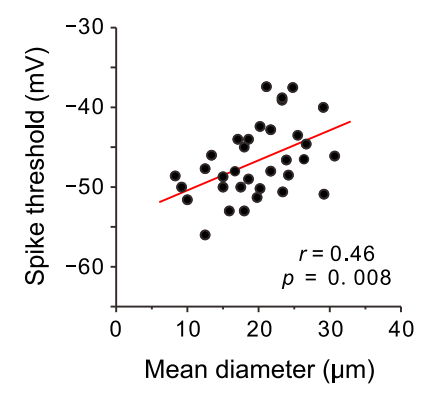

F

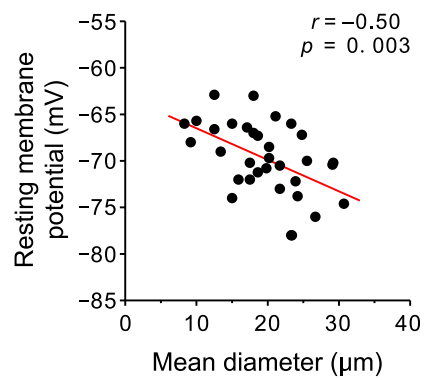

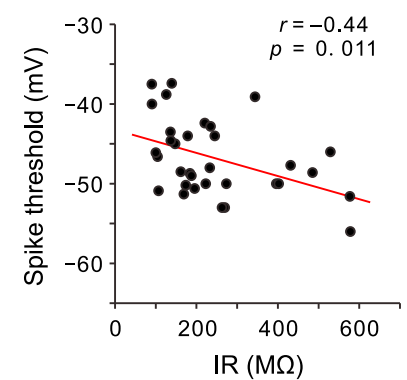

Figure 3. Relationships between the sizes of MNs in the dl-TMN and IRs, resting membrane potentials, or spike thresholds. Aa, Ba, Small $(19 \times 17 \mu \mathrm{m})$ and large $(30 \times 21 \mu \mathrm{m})$ MNs filled with biocytin $(\boldsymbol{A a}$ and $\boldsymbol{B a}$, respectively). The minor and major axes are indicated with open and filled arrowheads, respectively. $\boldsymbol{A} \boldsymbol{b}, \boldsymbol{B} \boldsymbol{b}$, Membrane potential responses to depolarizing current pulses applied to small and large MNs at the resting membrane potential ( $\boldsymbol{A} \boldsymbol{b}$ and $\boldsymbol{B} \boldsymbol{b}$, respectively). $\boldsymbol{C}$, The inverse relationship between the sizes of MNs and IRs $(n=33)$. $\boldsymbol{D}$, The inverse relationship between the sizes of MNs and resting membrane potentials $(n=33)$. $\boldsymbol{E}$, The positive relationship between the sizes of MNs and spike thresholds $(n=33)$. $\boldsymbol{F}$, The inverse relationship between the IRs and spike thresholds $(n=33)$.

following composition (in mM): $123 \mathrm{~K}$-gluconate, $8 \mathrm{KCl}, 20$ $\mathrm{NaCl}, 0.5 \mathrm{MgCl}_{2}, 2$ ATP-Na, 0.3 GTP-Na, 10 HEPES, and $0.1 \mathrm{EGTA}$; the $\mathrm{pH}$ was adjusted to 7.3 with $\mathrm{KOH}$. In some experiments, $123 \mathrm{~mm} \mathrm{~K}$-gluconate, $8 \mathrm{~mm} \mathrm{KCl}$, and $20 \mathrm{~mm} \mathrm{NaCl}$ were substituted for $118 \mathrm{~mm} \mathrm{~K}$-gluconate, 18 $\mathrm{mm} \mathrm{KCl}, 14 \mathrm{~mm} \mathrm{NaCl}$, and $10 \mathrm{~mm}$ biocytin to characterize the morphology of MNs. The patch pipettes had a DC resistance of 4-5 M $\Omega$ when filled with the internal solution. The membrane potential values given in the text were corrected for the junction potential between the internal solution (negative) and the standard extracellular solution $(10 \mathrm{mV})$. All recordings were made at room temperature $\left(20-24^{\circ} \mathrm{C}\right)$. The sealing resistance was usually $>10 \mathrm{G} \Omega$. Whole-cell currents or voltages were low-pass filtered at 2 $\mathrm{kHz}$ (four-pole Bessel filter) and digitized at a sampling rate of $10 \mathrm{kHz}$ (Digidata 1322A, MDS Analytical Technologies). Under the current-clamp condition, depolarizing and hyperpolarizing current pulses were applied every 30 $\mathrm{s}$. The IR was calculated from the $I-V$ relationships, which were usually linear when measured at the peak timing of the hyperpolarizing responses or sag potentials evoked immediately after the onset of negative current pulses (Fig. 6Aa, blue circle). Microstimulation of $100 \mu$ s duration was delivered via a sharp monopolar tungsten electrode (DC resistance, $1 \mathrm{M} \Omega$ ), which was placed just dorsal to or in the dorsal part of the TMN to simulate the presumed la input arising from the mesencephalic trigeminal sensory neurons (Fig. 4Aa). The united or stem axons of the mesencephalic trigeminal sensory neurons, which bifurcate into the peripheral axons innervating muscle spindles and the central axons projecting into the TMN, are located just dorsal to the TMN (Shigenaga et al., 1988, 1990). The intensity of the stimulus current applied to the dorsal part of the TMN was $<5 \mu \mathrm{A}$. The direct current spread is very confined within a small area as the amplitude of the maximum response evoked at a site around the tip of the stimulating electrode immediately after stimulation sharply decreases by half at a distance of $80 \mu \mathrm{m}$ and to almost zero at $<200 \mu \mathrm{m}$ from the tip of the stimulating electrode in slices (Sato et al., 2008). Thus, the stimulus current does not spread over the entire dl-TMN $(500 \times$ $1000 \mu \mathrm{m}$ ). 8-Br-cGMP (a membrane permeable analog of cGMP, which is the second messenger of NO; SigmaAldrich) was bath applied at a concentration of $100 \mu \mathrm{M}$.

\section{Preparation of Xenopus oocytes expressing TASK1 and TASK 3 channels}

The isolation and maintenance of the oocytes of frogs (Xenopus laevis) and injection with cRNA were performed as described previously (Inanobe et al., 2001). Mouse TASK1 and TASK3 cDNAs were obtained from pIRES2-ZsGreen1-TASK1 (Toyoda et al., 2010) and pIRES2-ZsGreen1-TASK3, respectively. Mouse TASK1 and TASK3 cDNAs were subcloned into pcDNA3.1(-) vector under the control of the $T 7$ promotor and 

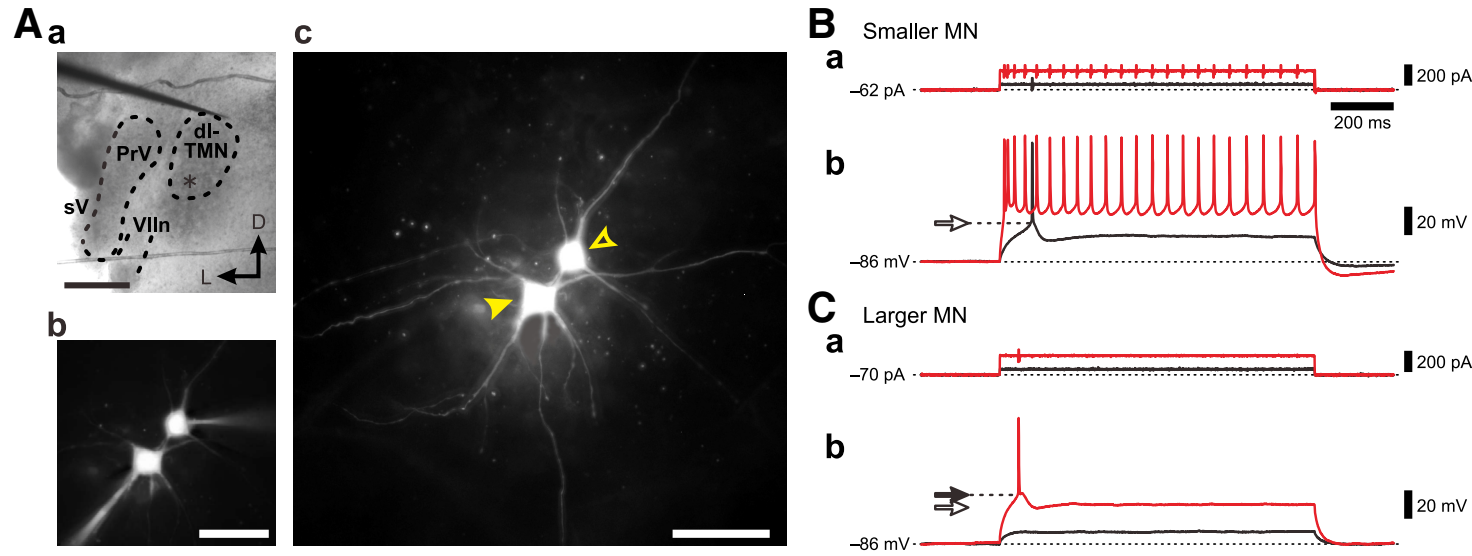

Figure 4. Membrane potential responses to depolarizing current pulses in smaller and larger MNs in the dl-TMN. Aa, A brainstem slice including the dl-TMN. A stimulation electrode was placed just dorsal to the TMN. PrV, Trigeminal principle nucleus; VIIn, facial nerve; $\mathrm{sV}$, sensory root of the trigeminal nerve. Scale bar, $500 \mu \mathrm{m}$. A $\boldsymbol{b}$, A simultaneous recording obtained from a pair of smaller and larger MNs that were filled with Lucifer yellow. Scale bar, $100 \mu \mathrm{m}$. Ac, A Lucifer yellow image of the smaller and larger MNs after fixation with paraformaldehyde (open and filled arrowheads, respectively). Scale bar, $100 \mu \mathrm{m}$. $\boldsymbol{B}, \boldsymbol{C}$, Membrane potential responses to depolarizing current pulses applied to the smaller and larger MNs ( $\boldsymbol{B} \boldsymbol{b}$ and $\boldsymbol{C} \boldsymbol{b}$, respectively) from the same baseline membrane potential of $-86 \mathrm{mV}$ brought about by negative DC current injection of -62 and $-70 \mathrm{pA}$, respectively (Ba and $\mathbf{C a}$, respectively). The apparent spike threshold was lower in the smaller MN (open arrow) than in the larger MN (filled arrow).

were subjected to cRNA preparation. Straight-chain DNAs that were obtained by truncating the downstream sequences of circular plasmids of TASK1 and TASK3 were used as templates. The cRNAs for injection into oocytes were prepared with T7 RNA polymerase (Invitrogen). Oocytes were injected with cRNAs for TASK1 (50 ng/ oocyte) and TASK3 (50 ng/oocyte). After injection, oocytes were incubated at $18^{\circ} \mathrm{C}$ in ND96 solution, which contained the following (in $\mathrm{mm}$ ): $96 \mathrm{NaCl}, 2 \mathrm{KCl}, 1.8 \mathrm{CaCl} 2$, $1 \mathrm{MgCl}_{2}$, and 5 HEPES, pH 7.4 with $\mathrm{NaOH}$, and supplemented with gentamicin $(50 \mu \mathrm{g} / \mathrm{ml})$. Currents were recorded 1-3 d after the cRNA injection.

\section{Two-electrode voltage-clamp recordings from Xenopus oocytes}

Membrane currents of oocytes were recorded by twoelectrode voltage clamp using the GeneClamp 500 amplifier (MDS) at room temperature. Data were reproduced and analyzed with pCLAMP version 10 (MDS) and Clampfit version 10.2 (MDS). The bath solution contained the following (in $\mathrm{mM}$ ): $90 \mathrm{KCl}, 3 \mathrm{MgCl}_{2}, 0.15$ niflumic acid, and 5 HEPES, $\mathrm{pH} 7.4$ with $\mathrm{KOH}$. The tip resistance of the glass electrodes was $0.4-1.5 \mathrm{M} \Omega$ when filled with the $3 \mathrm{M} \mathrm{KCl}$ pipette solution. Niflumic acid (0.1 mm; Sigma-Aldrich) was added in the bath solution to block $\mathrm{Ca}^{2+}$-dependent $\mathrm{Cl}^{-}$channels, which are richly expressed in the plasma membrane of oocytes (Dascal, 1987). 8-Br-cGMP was bath applied at concentration of $100 \mu \mathrm{M}$. The currents were measured $5-10 \mathrm{~ms}$ before the offset of the voltage step.

\section{Optical recording using $\mathrm{RH} 414$}

The procedures for the optical recording and its data analysis were previously described (Sato et al., 2008). Briefly, the slices were stained with $200 \mu \mathrm{M} \mathrm{N}$-(3triethylammoniumpropyl)-4-(4-(4- (diethylamino) phenyl) butadienyl) pyridinium dibromide ( $\mathrm{RH} 414$, Molecular Probes; Grinvald et al., 1984). The stained slices were illuminated by light with a wavelength of $535 \pm 15 \mathrm{~nm}$ using a stabilized $150 \mathrm{~W}$ xenon lamp. The fluorescence emitted from RH414 was long-pass filtered above $580 \mathrm{~nm}$ and measured with a CCD camera (NeuroCCD-SM, RedShirtlmaging), which was attached to a microscope (BX-51WI, Olympus) equipped with a water-immersion objective (10×, 0.3 numerical aperture; Olympus). The imaged area was $1.6 \times 1.6 \mathrm{~mm}^{2}$, and each pixel (element) of the $80 \times 80$ array detected the optical signals generated by a square region $\left(20 \times 20 \mu \mathrm{m}^{2}\right)$. The fluorescence images were captured at a sampling rate of $1000 \mathrm{~Hz}$. Repetitive electrical stimulation (10 pulses at $100 \mathrm{~Hz}$ ) of $100 \mu \mathrm{s}$ duration was delivered via a sharp monopolar tungsten electrode (DC resistance, $1 \mathrm{M} \Omega$ ) placed just dorsal to or in the dorsal part of the TMN to simulate presumed la afferents (Fig. 9A). 8-Br-cGMP was bath applied at a concentration of $100 \mu \mathrm{M}$. The amplitudes of the 1st to 9th (before 8-Br-cGMP application) or the 10th (after 8-Br-cGMP application) optical responses at the region of interest placed in the dl-TMN (Fig. 9A) were normalized to that in the 10th response obtained before the application of 8-Br-cGMP (Fig. 9D).

\section{Data analysis}

Statistical analysis was performed using STATISTICA10J (StatSoft). Numerical data were expressed as the mean \pm SD. The statistical significance was assessed using ANOVA followed by Fisher's PLSD post hoc test, paired and unpaired Student's $t$ test, Pearson's correlation coefficients, Wilcoxon signed rank test, and Wilks' lambda. The Student's $t$ test was used when the data showed a normal distribution, as confirmed by the KolmogorovSmirnov test. Statistical results are given as a $p$ value, 
except when it is very small $(p<0.001)$. A $p$ value $<0.05$ was considered statistically significant.

\section{Results}

The expression levels of TASK1 and TASK3 mRNAs in small and large MNs in the dl-TMN

First, we quantified the expression levels of TASK1 and TASK3 mRNAs in small $(15-20 \mu \mathrm{m})$ and large $(>35 \mu \mathrm{m})$ MNs in the dl-TMN using a real-time PCR method. The representative example of quantitative PCR for TASK1, TASK3, and GAPDH mRNAs obtained from 80 large MNs and that obtained from 400 small MNs are shown in Figure 1, $\mathrm{Ca}$ and $\mathrm{Cb}$. Under the condition that the PCR amplification efficacy was the same between the TASK1 and TASK3 primers (see Materials and Methods), the expression level of TASK1 mRNA was estimated to be two times higher than that of TASK3 mRNA in large MNs $(p<0.001$, paired $t$ test; $n=7$ samples), while the former was 20 times higher than the latter in small MNs $(p<$ 0.001 , paired $t$ test; $n=7$ samples; Fig. $1 D$ ).

As revealed by the relationship between the cell number and the $\mathrm{C}_{\mathrm{T}}$ of GAPDH mRNA (Fig. $1 E$ ), the mean $\mathrm{C}_{\mathrm{T}}$ value obtained from 50 or 400 small MNs was almost the same or very slightly smaller than that obtained from 10 or 80 large MNs, respectively, while that obtained from 200 small MNs was slightly larger than that obtained from 50 large MNs. This suggests that the GAPDH content in a single large $\mathrm{MN}$ is at least more than four times and very close to five times larger than that in a single small MN. This value is consistent with the volume ratio between the mean cell volumes of the small and large MNs: compared to the volume ratio ( $>5.4$ times) of large spheres to small ones (diameter ratio $>35 / 20$ ), the volume ratio of large polygonal neurons to small ones can be smaller, depending on their shapes $(35 / 20>$ axis ratio $>[35 \times \cos$ $\left.\left(\theta_{1}\right)\right] /\left[20 \times \cos \left(\theta_{2}\right)\right]$, when $\theta_{1}$ is larger than $\theta_{2} ; 0<\theta<90^{\circ}$ ), given that polygonal shape is a quadrangular pyramid. Based on the assumption that GAPDH content in a single large $\mathrm{MN}$ is five times larger than that in a single small $\mathrm{MN}$, we estimated the expression levels of TASK1 and TASK3 mRNAs in single large and small MNs. The expression level of TASK1 mRNA in one large MN was estimated to be 2.1 times higher than that in one small $\mathrm{MN}(p<0.001$, unpaired $t$ test; $n=7$ samples), while that of TASK3 mRNA in one large MN was estimated to be 20 times higher than that in one small MN $(p<0.001$, unpaired $t$ test; $n=7$ samples; Fig. 1F), suggesting that the IR in one large $\mathrm{MN}$ is much smaller than that in one small MN.

\section{Subcellular distributions of TASK1 and TASK3 channels in MNs in the dl-TMN}

As we made a new antibody to TASK1 in the present study (see Materials and Methods), we first examined its selectivity using HEK293 cells transfected with TASK1 or mock together with ZsGreen1. In the HEK293 cells represented by DAPI staining (Fig. $2 A b, B b$ ), TASK1 or mock transfection was ensured by ZsGreen1 expression (Fig. $2 A c, B c)$. In the HEK293 cells transfected with TASK1, the strong TASK1 immunoreactivity was seen along the plasma membrane and partly in the cytoplasm (Fig. 2Aa), while the HEK293 cells transfected with mock displayed no TASK1 immunoreactivity (Fig. 2Ba). These results suggest that this antibody is specific to the TASK1 protein heterologously expressed in HEK cells.

We then examined TASK1 immunoreactivity in MNs in the dl-TMN. In ChAT-positive MNs, their somata were strongly immunoreactive to anti-TASK1 antibody (Fig. 2C, filled arrowheads), whereas their neuropils were very weakly immunoreactive to the antibody (Fig. 2C). When the anti-TASK1 antibody was absorbed by preincubating with the antigen peptide of TASK1, the TASK1 immunoreactivity was seen neither in TMN (Fig. $2 D$ ) nor in the other brain regions where TASK1 immunoreactivity was seen, as follows. The distribution pattern of TASK1 immunoreactivity was found to be remarkably similar to that demonstrated using in situ hybridization in previous studies (Karschin et al., 2001; Talley et al., 2001). Strong TASK1 immunoreactivity was found not only in the trigeminal MNs but also in hypoglossal and facial MNs. The moderate TASK1 immunoreactivity to this antibody was found in neurons in barrel and insular cortices, cerebellar granule layers, the nucleus of solitary tract, and intermediate reticular nucleus, while the spinal tract nucleus of trigeminal nerve and superior cerebellar peduncle displayed weak immunoreactivity. Thus, there were three lines of evidence that ensured the specificity of the TASK1 antibody. In contrast, somata of ChAT-positive MNs were almost immunonegative to anti-TASK3 antibody (Fig. 2E, filled arrowheads), while their neuropils showed intense TASK3 immunoreactivity (Fig. 2E). Thus, TASK1 immunoreactivity was seen almost exclusively in the somata, while TASK3 immunoreactivity was seen only in dendrites instead of the somata, as shown in the middle panels of Figure 2, $C$ and $E$. The merged images clearly show that the somata, instead of the dendrites, are shown as yellow (i.e., green plus red) in the bottom panel of Figure $2 \mathrm{C}$, while the dendrites, instead of the somata, are shown as yellow (i.e., green plus red) in the bottom panel of Figure $2 E$. These results clearly indicate that TASK1 and TASK3 are complementarily expressed in somata and dendrites, respectively, regardless of the size of the MNs in the dl-TMN.

\section{Inverse relationship between the size of MNs in the dI-TMN and IRs}

We next investigated the relationships between the sizes of $\alpha \mathrm{MNs}$ in the dl-TMN and IRs, resting membrane potentials, or spike thresholds. Whole-cell current-clamp recordings were obtained from $33 \mathrm{MNs}$ that displayed polygonal and stellate-shaped somata with many primary dendrites when observed under Nomarski optics $(63 \times$; Fig. 3). The membrane potential responses were evoked by the injection of depolarizing and hyperpolarizing current pulses at resting membrane potentials (Fig. $3 A b, B b$ ). After the recordings, MNs filled with biocytin were fixed with $4 \%$ paraformaldehyde, and the sizes of the labeled neurons were evaluated with a confocal microscope image (Fig. $3 A a, B a)$. The morphological features of these multipolar $\alpha \mathrm{MNs}$ were distinct from those of $\gamma \mathrm{MNs}$, which 
show piriform or round-shaped somata with fewer primary dendrites (Card et al., 1986; Bae et al., 2002).

The IRs and resting membrane potentials were inversely correlated with the sizes of $\mathrm{MNs}$, as revealed by their significant negative correlations (Fig. $3 C, D$, respectively), while the spike threshold was significantly positively correlated with the sizes of MNs (Fig. 3E). Subsequently, the spike threshold was significantly inversely correlated with the sizes of MNs (Fig. 3F). These results indicate that smaller MNs in the dl-TMN have larger IRs, depolarized resting membrane potentials, and lower spike thresholds, while larger MNs in the dl-TMN have smaller IRs, hyperpolarized resting membrane potentials, and higher spike thresholds. In the next series of experiments, we examined whether MNs in the dl-TMN are recruited based on their soma sizes or IRs using simultaneous whole-cell current-clamp recordings from a pair of MNs of different size.

\section{The orderly activation of MNs in the dl-TMN}

In the dl-TMN, simultaneous whole-cell recordings were obtained from a pair of randomly selected MNs that are located close each other to avoid the differences in the numbers of synaptic inputs and postsynaptic receptors in the slice preparations, one of which is relatively larger or smaller than the other (Fig. 4Ab,Ac, open and filled arrows, respectively). In such a pair of MNs, membrane potential responses to the depolarizing current pulses were examined at the same baseline membrane potential of $-86 \mathrm{mV}$ brought about by negative DC current injections of -62 and $-70 \mathrm{pA}$, respectively (Fig. $4 \mathrm{Ba}, \mathrm{Ca}$, respectively). When a depolarizing current pulse with the same small intensity was applied to the pair of smaller and larger MNs (Fig. 4Ba,Ca, black traces), a spike was evoked only in the smaller MN (Fig. 4Bb, black trace) but not in the larger MN (Fig. 4Cb, black trace). When a depolarizing current pulse with the same large intensity was applied to the pair of the smaller and larger MNs (Fig. $4 B a, C a$, red traces), a repetitive spike firing was evoked in the smaller MN (Fig. $4 B b$, red trace), while only a single spike was evoked in the larger MN (Fig. 4Cb, red trace). The spike threshold in the smaller $\mathrm{MN}$ was apparently lower than that in the larger MN (Fig. 4Bb,Cb, compare open arrows, filled arrows).

We further compared their responses to hyperpolarizing current pulses and repetitive electrical stimulation at 100 $\mathrm{Hz}$, which was applied to the area just dorsal to the TMN, between the smaller and larger MNs. The responses to hyperpolarizing current pulses were apparently larger in the smaller MN than in the larger MN, suggesting that the IR in the smaller MN was larger than that in the larger MN (Fig. 5A,B). As the stimulus intensity was increased, EPSPs evoked by $100 \mathrm{~Hz}$ stimulation increased in amplitude in both the smaller and larger MNs, and were temporally summated to consequently trigger spikes. However, the first spiking invariably occurred at an earlier time in the smaller MN than in the larger MN, regardless of stimulus intensity (Fig. 5C,D, red, orange arrowheads). The spike threshold in the smaller MN was apparently lower than that in the larger MN (Fig. 5C,D, compare open arrows, filled arrows), consistent with the observation made in response to the depolarizing current pulses (Fig. 4B,C).

The pooled data analysis of the simultaneous wholecell recordings obtained from five pairs of MNs of different sizes revealed that the latency to the first spike was invariably shorter in a smaller MN than in a larger MN (Fig. $5 E)$ and that the spike threshold was invariably lower in a smaller MN than in a larger MN (Fig. 5F). The median of the latency to the first spike obtained from smaller MNs (22 ms) was significantly shorter $(p=0.043$, Wilcoxon signed rank test) than that obtained from larger MNs (32.8 ms; Fig. 5E). The median of the spike threshold obtained from smaller MNs $(-57.7 \mathrm{mV})$ was significantly lower $(p=$ 0.043 , Wilcoxon signed rank test) than that obtained from larger MNs (-52.8 mV; Fig. 5F). Given the pair recordings from a neuronal population in which the inverse relationships were observed between IRs and latencies to the first spikes or spike thresholds, these results suggest that MNs in the dl-TMN are recruited in an orderly manner, depending on their sizes or IRs.

It has been shown previously that TASK1-mediated leak $\mathrm{K}^{+}$currents in basal forebrain cholinergic neurons were activated by NO signaling through the cGMP/PKG transduction pathway (Toyoda et al., 2010). Cholinergic neurons located in the pedunculopontine and laterodorsal tegmentum nuclei and the ventromedial medullary reticular formation are known to be a possible source of nitrergic input to the TMN (Pose et al., 2005; Travers et al., 2005). Thus, it is possible that NO modulates TASK channels expressed in the MNs in the dl-TMN.

\section{Effects of 8-Br-cGMP on IRs in small and large MNs in the dl-TMN}

Under the current-clamp condition, $100 \mu \mathrm{M}$ 8-Br-cGMP hyperpolarized the membrane potential and reduced the number of spikes evoked by a depolarizing current pulse in a small MN (Fig. $6 A a, A b)$. Then, $I-V$ relationships were measured at the peak timing of sag potentials and just before the offset of current pulses (see Materials and Methods). An inwardly rectifying current $\left(I_{\text {ir }}\right)$ became apparent at potentials more negative than $-100 \mathrm{mV}$ as represented by an inwardly rectifying $I-V$ relationship measured just before the offset of current pulses (Fig. $6 A c$, blue crosses), in contrast to the almost linear $I-V$ relationship measured at the peak timing of sag potentials (Fig. 6Ac, blue circles). However, the slope of the linear regression line for the $I-V$ relationship measured at potentials ranging between the resting membrane potential and $-100 \mathrm{mV}$ was almost the same as between those measured at the peak timing of sag potentials and before the offset of current pulses (Fig. 6Ac). From this rectifying potential at approximately $-100 \mathrm{mV}$, it is also suggested that the $I_{\text {ir }}$ is mediated by a voltage-dependent $\mathrm{K}^{+}$current, instead of an h current. Thus, neither $I_{\text {ir }}$ nor currents other than voltage-independent leak $\mathrm{K}^{+}$currents are involved in the IR that was measured at the peak of sag potentials evoked in MNs of dl-TMN in response to the hyperpolarizing current pulses applied at the resting membrane potential. 
A
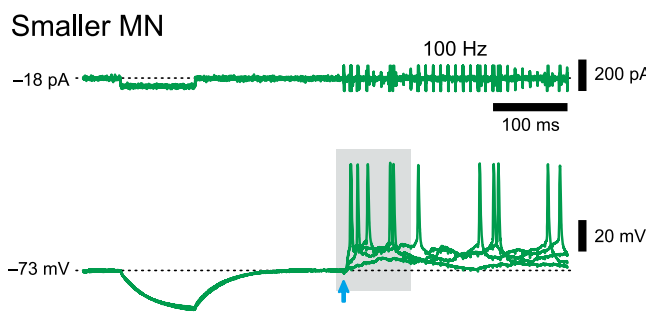

\section{B Larger MN}
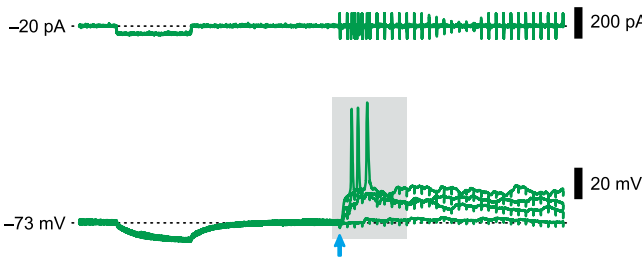

\section{E}

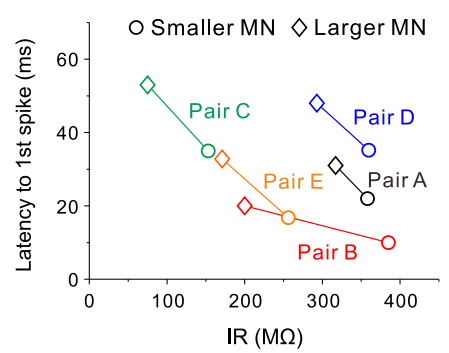

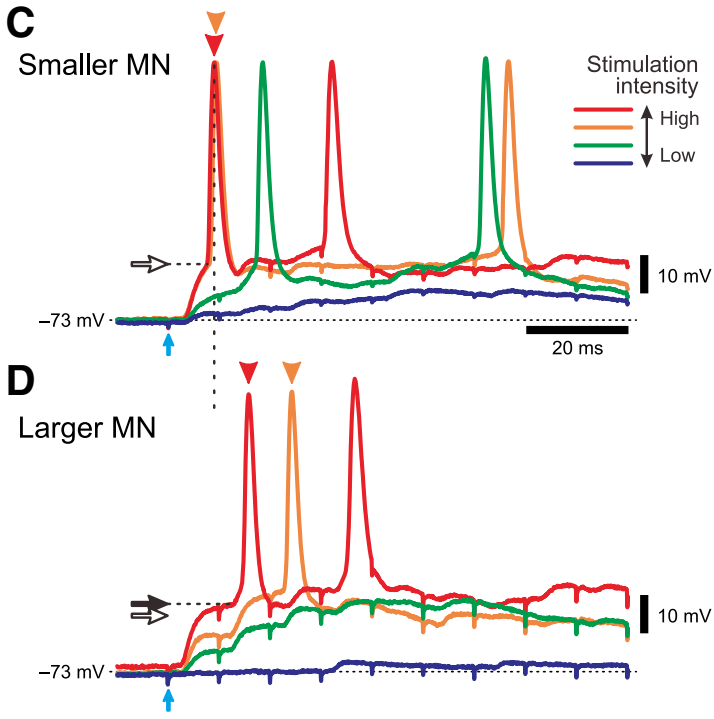

F

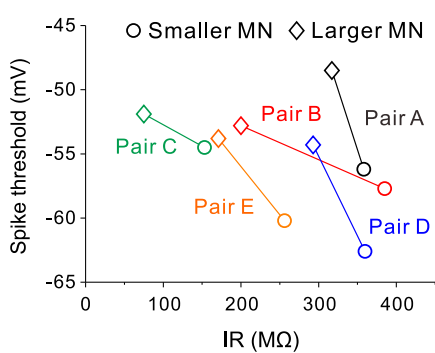

Figure 5. IR-dependent activation/recruitment of MNs in the dl-TMN. A, B, Membrane potential responses to hyperpolarizing current pulses and those evoked by $100 \mathrm{~Hz}$ stimulation of presumed la afferents in the smaller and larger MNs (A and $\boldsymbol{B}$, respectively). $\boldsymbol{C}, \boldsymbol{D}$, The enlarged traces of the responses (gray area in $\boldsymbol{A}$ and $\boldsymbol{B}$, respectively) in the smaller and larger MNs (C and $\boldsymbol{D}$, respectively). $\boldsymbol{E}$, The relationship between IRs and latencies to the first spikes in five pairs of MNs of different sizes when the difference in the latency to the first spike between the pair of smaller and larger MNs became maximum. $\boldsymbol{F}$, The relationship between IRs and spike thresholds in five pairs of MNs of different sizes.

Accordingly, the $I-V$ relationships that were measured at peak sag potentials were compared before and after the application of 8-Br-cGMP (Fig. 6Ac, blue circles, red circles). The slope of the linear regression line for the $I-V$ relationship obtained after 8 -Br-cGMP application became less steep compared with that obtained before 8-Br-cGMP application, and the two regression lines crossed at approximately $-95 \mathrm{mV}$ (the equilibrium potential for $\mathrm{K}^{+}$). This observation clearly indicates that 8-BrcGMP decreased the IR through an enhancement of leak $\mathrm{K}^{+}$currents without causing contamination by other conductances. In contrast, the inwardly rectifying component was not markedly affected by 8-Br-cGMP (Fig. 6Ac, blue crosses, red crosses), suggesting that at potentials more hyperpolarized than $-100 \mathrm{mV}$, where $I_{\text {ir }}$ was activated, the enhancement of leak $\mathrm{K}^{+}$current by 8 -Br-cGMP was largely masked by $I_{\mathrm{ir}}$.

In a large MN, 8-Br-cGMP slightly depolarized the membrane potential and increased the number of spikes (Fig. $6 B a, B b$ ). The slope of the linear regression line for the $I-V$ relationship obtained after $8-B r-c G M P$ application became slightly steeper compared with that obtained before 8-Br-cGMP application, and the two regression lines crossed at approximately $-95 \mathrm{mV}$, indicating a slight increase in the IR due to an inhibition of leak $\mathrm{K}^{+}$currents (Fig. $6 \mathrm{BC}$ ). These results strongly suggest that $8-\mathrm{Br}-\mathrm{cGMP}$ changed membrane properties differentially between small and large MNs through differential modulation of leak $\mathrm{K}^{+}$currents. The pooled data analysis in $14 \mathrm{MNs}$ revealed that 8-Br-cGMP decreased the IRs in MNs that have larger IRs, while it either hardly changed or slightly increased those in MNs that have smaller IRs (Fig. 6C). Subsequently, the changes in IRs by 8 -Br-cGMP were inversely correlated with the IRs of MNs, as revealed by the significant negative correlation $(p<0.001, r=$ -0.89 , Pearson's correlation) between the two parameters (Fig. 6C).

The effects of 8-Br-cGMP on IRs in large MNs were unexpected because TASK1 channels were more largely expressed in large MNs compared with small MNs (Fig. 1). However, if TASK3 channels expressed in the dendrites of large MNs are suppressed by $8-\mathrm{Br}$ cGMP, it is possible that 8-Br-cGMP does not necessarily change IRs in large MNs, presumably due to the opposing effects of 8-Br-cGMP on TASK1 and TASK3 channels. If this is the case, the IRs of dendritic membrane in large MNs would be increased through the suppression of TASK3 channels. To examine this pos- 

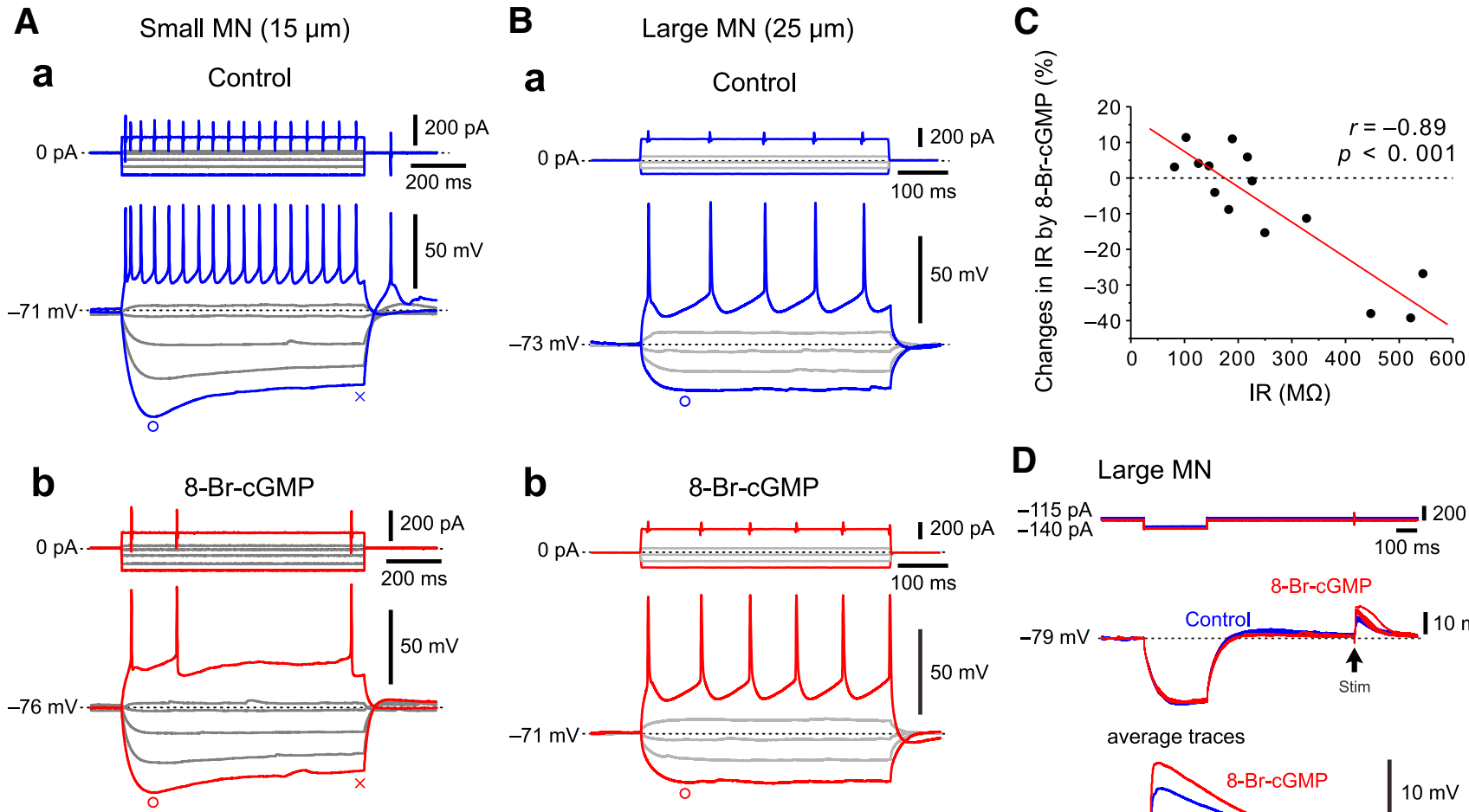

\section{Large $\mathrm{MN}$}
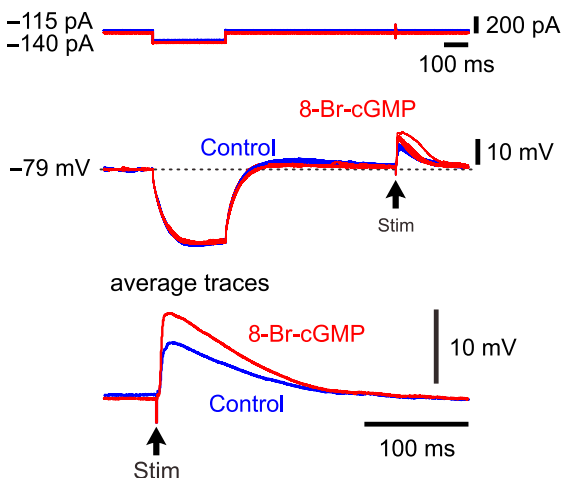

C

Current (pA)

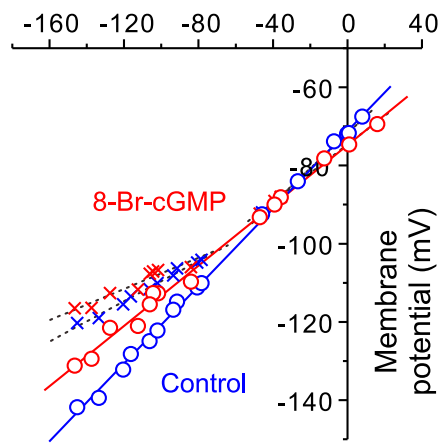

C

Current (pA)

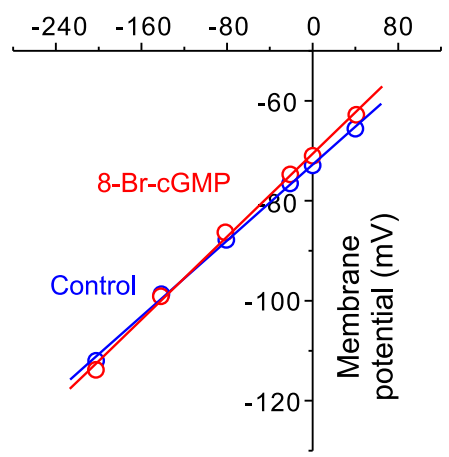

E

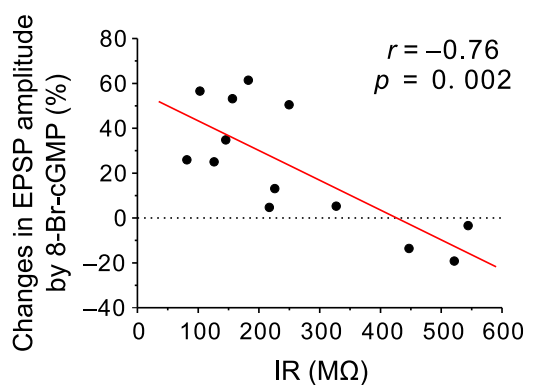

Figure 6. Modulation of IRs by 8-Br-cGMP in small and large MNs in the dl-TMN. $\boldsymbol{A a}, \boldsymbol{A b}$, Sample traces of responses to depolarizing and hyperpolarizing current pulses obtained before application $(\boldsymbol{A a})$ and during application $(\boldsymbol{A} \boldsymbol{b})$ of $8-\mathrm{Br}-\mathrm{cGMP}$ in a small MN. $\boldsymbol{A} \boldsymbol{c}$, The $I-V$ relationships obtained before and during application of $8-B r-c G M P$ in a small MN. The $I-V$ relationships measured at the peak sag potentials are almost linear (blue and red circles), while those measured just before the offset of current pulses are inwardly rectifying (blue and red crosses). $\mathbf{B a}, \mathbf{B b}$, Sample traces of responses to depolarizing and hyperpolarizing current pulses obtained before application $(\mathbf{B a})$ and during application $(\mathbf{B b})$ of $8-\mathrm{Br}$-cGMP in a large MN. $\mathbf{B c}$. The $I-V$ relationships obtained before and during the application of 8-Br-cGMP in a large MN. C , The relationship between IRs of MNs and changes in IRs by $8-B r-c G M P(n=14)$. $\boldsymbol{D}$, EPSPs evoked by stimulation just dorsal to the TMN obtained before and during the application of 8-Br-cGMP in a large MN. $\boldsymbol{E}$, The relationship between IRs and changes in EPSP amplitudes by 8-Br-cGMP $(n=13)$.

sibility, we next investigated the effects of 8 -Br-cGMP on EPSPs evoked by a single electrical stimulation applied just dorsal to or in the dorsal part of the TMN in large MNs. Indeed, the amplitudes of EPSPs evoked in larger MNs that have small IRs were increased after the application of $100 \mu \mathrm{M}$ 8-Br-cGMP (Fig. 6D; also see Fig. $6 E$ ), suggesting that TASK3 channels expressed in dendrites of large MNs are suppressed by 8 -Br-cGMP. In contrast, 8-Br-cGMP decreased the amplitudes of EPSPs in smaller MNs that have larger IRs (Fig. 6E). The pooled data analysis in $13 \mathrm{MNs}$ revealed that the changes in EPSP amplitude by 8 -Br-cGMP were significantly inversely correlated with the IRs of MNs (Fig. $6 E ; p=0.002$, $r=-0.76$, Pearson's correlation). Taken together, these results suggest that 8 - $\mathrm{Br}$-cGMP decreases IRs in smaller MNs in the dl-TMN due to the activation of TASK1 channels, while it either hardly changes or slightly increases those in larger MNs in the dl-TMN, presumably due to the opposing effects of 8-Br-cGMP on TASK1 and TASK3 channels (Fig. 8; Discussion). 
A Smaller MN

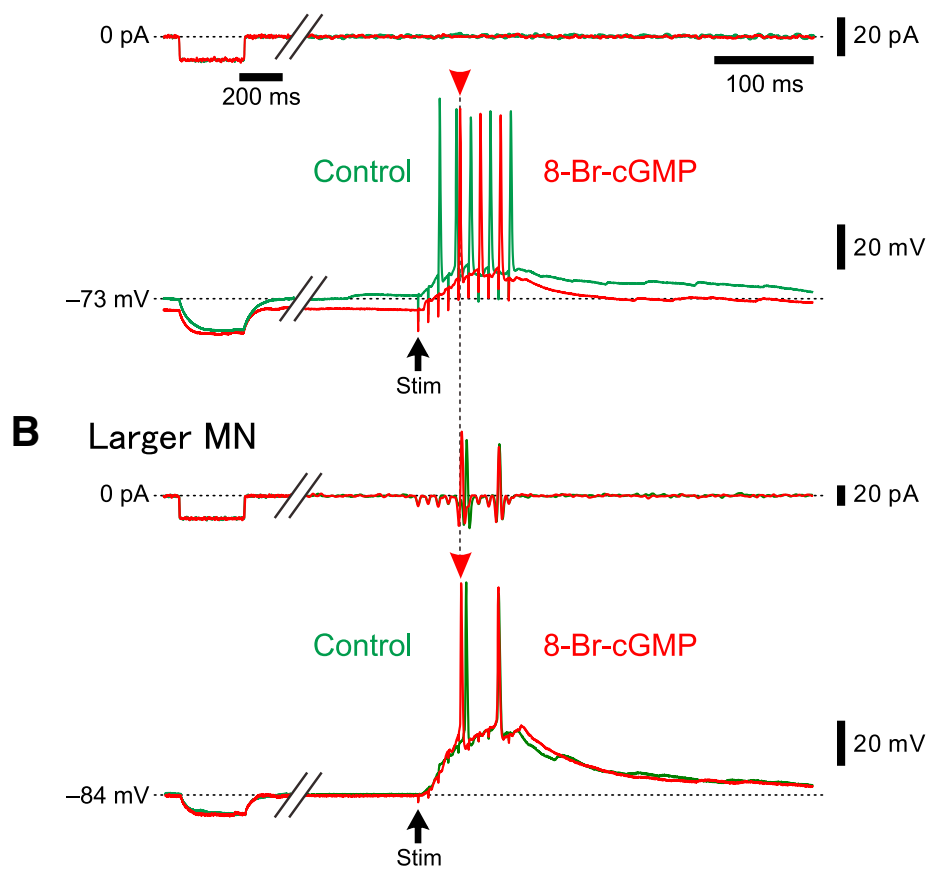

C

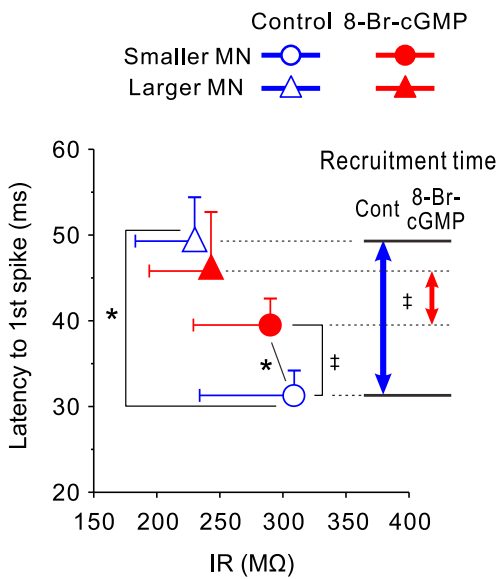

Figure 7. 8-Br-cGMP differentially modulates spike-onset latencies between smaller and larger MNs in the dl-TMN. $\boldsymbol{A}$, $\boldsymbol{B}$, Membrane potential responses to hyperpolarizing current injections and those evoked by $100 \mathrm{~Hz}$ stimulation of presumed la afferents in smaller and larger MNs ( $\boldsymbol{A}$ and $\boldsymbol{B}$, respectively) obtained before (green traces) and during the application of 8-Br-cGMP (red traces). $\boldsymbol{C}$, The relationship between the IRs and the latencies to first spikes obtained before and after the application of $8-\mathrm{Br}$-cGMP in four pairs of MNs of different sizes. The blue and red double-headed arrows represent the recruitment time, calculated as the difference in the mean latency to the first spike between the smaller and larger MNs obtained before and after the application of 8-Br-cGMP, respectively. $* p<0.05$, Wilks' lambda; $\neq p<0.05$, paired $t$ test.

\section{8-Br-cGMP modulates the latency to the first spike in MNs in the dl-TMN}

We next examined the effects of 8-Br-cGMP on the rankordered recruitment induced by repetitive electrical stimulation of la afferents in a pair of MNs in the dl-TMN, which were simultaneously recorded. 8-Br-cGMP decreased the IR and hyperpolarized the resting membrane potential, which consequently increased the latency to the first spike in a smaller MN (Fig. 7A, compare green traces, red traces). In contrast, 8 -Br-cGMP did not markedly change the IR or the resting membrane potential and slightly decreased the latency to the first spike in a larger MN (Fig. 7B). Consequently, this pair of MNs displayed almost the same latency to the first spike (Fig. $7 A, B$, compare arrowheads).

In the four pair recordings obtained from two MNs of different sizes that were visually identified under Nomarski optics, the IR and the latency to the first spike were measured before and after the application of 8-Br-cGMP and were plotted in the two-dimensional space defined by the IR and the latency to the first spike (Fig. 7C). The smaller MNs were invariably larger in the IR and shorter in the latency to the first spikes compared with the larger MNs in any pair recordings. The mean values of the IRs and the latencies to the first spikes were significantly $(p=$ 0.045 and $p=0.029$, respectively, paired $t$ test) different between the smaller and larger MNs.
The discrimination analysis revealed that 8 -Br-cGMP significantly ( $p=0.032$, Wilks' lambda) changed the distribution of the IR and the latency to the first spike in the two-dimensional space in smaller MNs (Fig. 7C, blue open circles, red filled circles). The mean value of the latencies to the first spike was significantly ( $p=0.045$, paired $t$ test) increased from $31.3 \pm 2.9$ to $39.5 \pm 3.1 \mathrm{~ms}$ following the application of 8-Br-cGMP (Fig. 7C), although there was no significant ( $p=0.129$, paired $t$ test) difference in the mean value of the IRs obtained before and after the application of 8-Br-cGMP (309 \pm 75 and $290 \pm 61 \mathrm{M} \Omega$, respectively). In contrast, in larger MNs, 8-Br-cGMP did not significantly $(p=0.676$, Wilks' lambda) change the distribution of the IR and the latency to the first spike (Fig. $7 C$, blue open triangles, red filled triangles). There were no significant differences either in the mean value of the IRs $(p=0.101$, paired $t$ test) or in the mean value of the latencies to the first spikes ( $p=0.140$, paired $t$ test) obtained before (230 $\pm 47 \mathrm{M} \Omega$ and $49.3 \pm 5.1 \mathrm{~ms}$, respectively) and after (243 $\pm 49 \mathrm{M} \Omega$ and $45.8 \pm 6.9 \mathrm{~ms}$, respectively) the application of 8-Br-cGMP.

Before the application of 8-Br-cGMP, the distribution of the IR and the latency to the first spike in smaller MNs were significantly ( $p=0.006$, Wilks' lambda) different from those in larger MNs (Fig. 7C, blue open circle and triangle). However, following the application of $8-\mathrm{Br}$ cGMP, there was no significant ( $p=0.298$, Wilks' lambda) 
difference in the distribution of the IR and the latency to the first spike between the smaller and larger MNs. Subsequently, the mean value of the difference in the latency to the first spike between the smaller and larger MNs was significantly ( $p=0.029$, paired $t$ test) decreased from 18.0 \pm 4.2 to $6.3 \pm 8.9 \mathrm{~ms}$ (Fig. $7 \mathrm{C}$, compare blue doubleheaded arrows, red double-headed arrows). These results suggest that $8-\mathrm{Br}$-cGMP decreases the time necessary for the recruitment starting with smaller MNs and progressing to larger MNs, causing a more synchronous activation of smaller and larger MNs in the dl-TMN.

\section{8-Br-cGMP inhibits TASK3 currents}

In small MNs that primarily express TASK1 channels (Figs. 1, 2), 8-Br-cGMP decreased the IRs (Fig. 6A,C). Because it has been previously shown that cloned TASK1 channels are activated by 8-Br-cGMP (Toyoda et al., 2010), it is strongly suggested that the decreases in IRs in small MNs are mediated by the activation of TASK1 channels. However, $100 \mu \mathrm{M}$ 8-Br-cGMP did not necessarily decrease IRs in large MNs (Fig. 6C) despite the higher expression of TASK1 channels in large MNs compared with small MNs (Fig. 1F). Because large MNs possess many dendrites that express TASK3 channels (Fig. 2E) and because 8-BrcGMP increased the amplitudes of EPSPs (Fig. 6E), it is possible that 8-Br-cGMP increases the IRs of dendritic membrane by inhibiting TASK3 channels. Therefore, we next investigated whether and how 8-Br-cGMP modulates cloned TASK3 channels that are heterologously expressed in Xenopus oocytes.

TASK3 currents were evoked by voltage steps ranging between -150 and $+60 \mathrm{mV}$ by $30 \mathrm{mV}$ steps applied at a holding potential of $-90 \mathrm{mV}$. When the extracellular $\mathrm{pH}$ was decreased from 8.4 to 7.4, TASK3 currents evoked in response to positive voltage steps ranging between 0 and +60 mV were markedly decreased (Fig. 8A,D). When 100 $\mu \mathrm{M}$ 8-Br-cGMP was bath applied, TASK3 currents in response to the positive voltage steps ranging between 0 and $+60 \mathrm{mV}$ at $\mathrm{pH} 7.4$ and 8.4 were markedly decreased (Fig. 8B,C, respectively; also see Fig. 8D). The pooled data analysis revealed that TASK3 currents at $0,+30$, and $+60 \mathrm{mV}$ were significantly decreased by 8 -Br-cGMP at $\mathrm{pH} 7.4(0 \mathrm{mV}, p=0.019 ;+30 \mathrm{mV}, p<0.001 ;+60 \mathrm{mV}, p$ $<0.001$, two-way ANOVA followed by Fisher's PLSD) and at $\mathrm{pH} 8.4(0 \mathrm{mV}, p=0.044 ;+30 \mathrm{mV}, p=0.001$; +60 mV, $p=0.001$, two-way ANOVA followed by Fisher's PLSD; Fig. $8 E, n=8$ ). As it has previously been reported (Toyoda et al., 2010) that 8-Br-cGMP enhances TASK1 currents heterologously expressed in HEK293 cells at pH 7.3, we confirmed that 8-Br-cGMP enhances TASK1 currents in Xenopus oocytes in which cloned TASK1 channels were heterologously expressed. TASK1 currents at $0,+30$, and $+60 \mathrm{mV}$ were significantly increased by $8-\mathrm{Br}-\mathrm{cGMP}$ at $\mathrm{pH}$ 7.4 (0 mV, $p=0.007$; +30 mV, $p=0.003$; +60 mV, $p<$ 0.001 , two-way ANOVA followed by Fisher's PLSD), but not at $\mathrm{pH} 8.4(0 \mathrm{mV}, p=0.228 ;+30 \mathrm{mV}, p=0.214 ;+60$ $\mathrm{mV}, p=0.161$, two-way ANOVA followed by Fisher's PLSD; Fig. $8 F, n=5$ ). Based on these results, it can be concluded that 8-Br-cGMP enhances TASK1 currents, whereas it suppresses TASK3 currents.

\section{Effects of 8-Br-cGMP on spatiotemporal patterns of excitation spreads in the dl-TMN}

We next investigated by using voltage-sensitive dye imaging whether and how spatiotemporal patterns of excitation spreads that are evoked by repetitive electrical stimulations (10 pulses at $100 \mathrm{~Hz}$ ) applied just dorsal to or in the dorsal part of the TMN (Fig. 9A) are modulated by 8-Br-cGMP. The excitation spread was gradually facilitated over the dl-TMN as the stimulation was repeated at $100 \mathrm{~Hz}$ (Fig. 9B). This frequency facilitation is characteristic of the la synapse on trigeminal MNs (Grimwood and Appenteng, 1995), in contrast to the well established frequency depression of la-EPSPs in spinal MNs (Curtis and Eccles, 1960). The temporal profile of the optical responses (Fig. 9D, control) revealed that the temporal summation of the optical responses occurred to recruit MNs in the dl-TMN. Following bath application of $100 \mu \mathrm{M}$ 8-Br-cGMP, the excitation spread in response to the first stimulation appeared to be decreased compared with the control responses (Fig. 9B,C, first stimulation), whereas the excitation spread in response to the 10th simulation appeared to be enhanced compared with the control response (Fig. 9B,C, 10th stimulation). As revealed by the averaged temporal profiles obtained before and after the application of 8-Br-cGMP in the five slices examined (Fig. 9D), 8-Br-cGMP decreased the peak amplitudes of early optical responses but increased those of late optical responses to 10 successive stimulations. The peak amplitude of optical responses induced by the first stimulation after application of 8-Br-cGMP $(0.61 \pm 0.12)$ was significantly smaller $(p=0.015$, two-way ANOVA followed by Fisher's PLSD) compared with that obtained before the application of $8-\mathrm{Br}$-cGMP (0.73 \pm 0.08$)$, while that induced by the 10th stimulation after the application of 8-Br-cGMP (1.13 \pm 0.04$)$ was significantly larger $(p=$ 0.007, two-way ANOVA followed by Fisher's PLSD) compared with that obtained before the application of $8-\mathrm{Br}$ cGMP (1; Fig. 9E, $n=5)$. Thus, 8-Br-cGMP suppressed early optical responses but facilitated late optical responses, indicating the inhibition of the earlier recruitment of smaller MNs and the facilitation of later recruitment of larger MNs, respectively, causing a more synchronous activation of small and large MNs in the dl-TMN. This is consistent with the effects of 8-Br-cGMP on the IRs of smaller and larger MNs and on EPSPs (Fig. 6), and also with the observations made by simultaneous whole-cell recordings from a pair of small and large MNs (Fig. 7). Thus, TASK channels play crucial roles in the rankordered recruitment of MNs in the dl-TMN.

\section{Discussion}

\section{TASK1/TASK3 mRNA expression levels and TASK1/ TASK3 subunit distribution in small and large MNs in the dl-TMN}

The immunohistochemistry revealed that TASK1 and TASK3 channels were complementarily distributed in soma and dendrites of MNs, respectively (Fig. 2), suggesting that there are few heterodimeric TASK1/3 channels in MNs in the dl-TMN. Then, the IR of MNs in the dl-TMN is likely to be determined primarily by the expres- 


\section{TASK3}

A
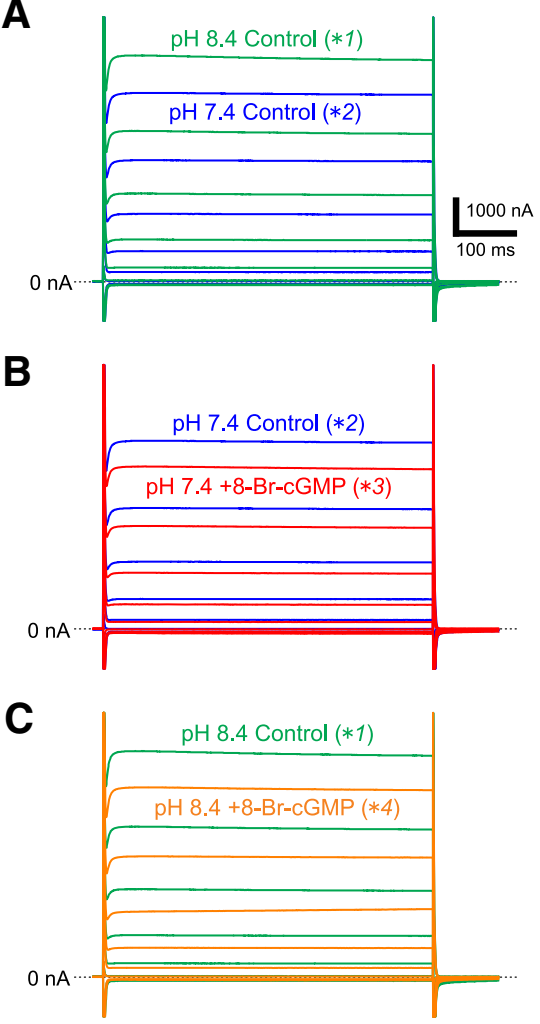

D

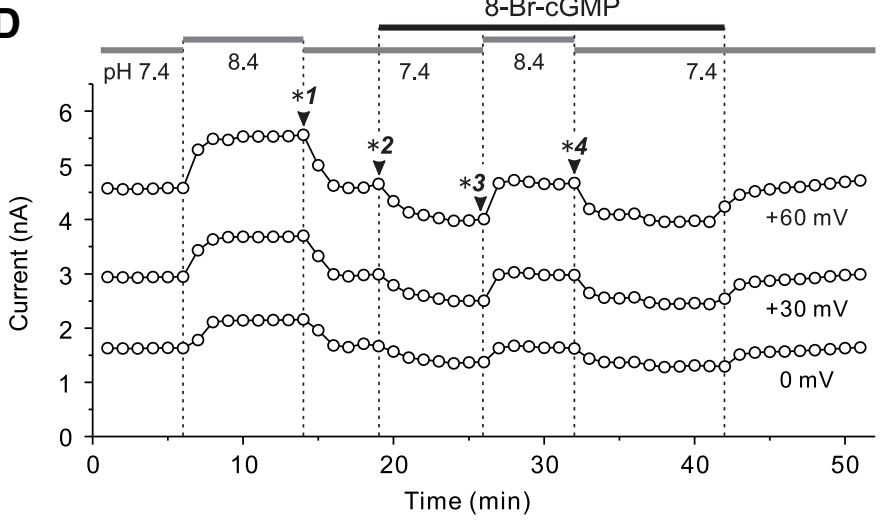

F

TASK3

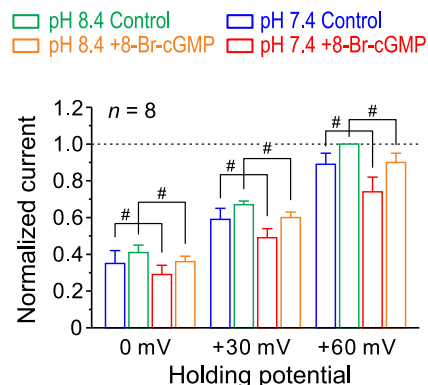

TASK1

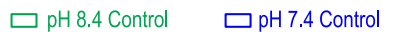

$\square \mathrm{pH} 8.4$ +8-Br-cGMP $\square \mathrm{pH} 7.4$ +8-Br-cGMP

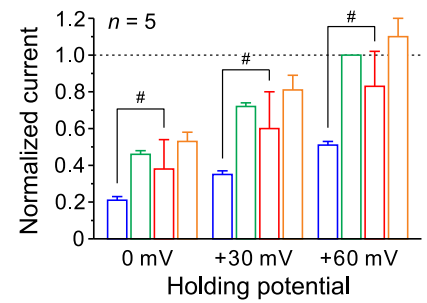

Figure 8. 8-Br-cGMP inhibits cloned TASK3 channels. $\boldsymbol{A}$, Superimposed TASK3 currents evoked by voltage steps ranging between -150 and $+60 \mathrm{mV}$ in $30 \mathrm{mV}$ steps applied at a holding potential of $-90 \mathrm{mV}$ at $\mathrm{pH} 8.4$ and 7.4. The respective current traces were obtained at the time points indicated with $* 1$ and $* 2$ in $\boldsymbol{D}$. $\boldsymbol{B}$, Superimposed TASK3 currents obtained at $\mathrm{pH} 7.4$ before (blue traces) and during (red traces) the application of $8-\mathrm{Br}$-cGMP. The respective current traces were obtained at the time points indicated with $* 2$ and $* 3$ in $\boldsymbol{D}$. C , Superimposed TASK3 currents obtained at pH 8.4 before (green traces) and during the application of 8-Br-cGMP (orange traces). The respective current traces were obtained at the time points indicated with $* 1$ and $* 4$ in $\boldsymbol{D}$. $\boldsymbol{D}$, Plots of TASK3 currents at $0,+30$, and $+60 \mathrm{mV}$ against time. Gray horizontal bars represent the timing and duration of perfusion of the extracellular solutions at respective $\mathrm{pH}$, and the black horizontal bar represents the timing and duration of the addition of $100 \mu \mathrm{M} 8-\mathrm{Br}$-cGMP. Arrowheads indicate the time points at which the respective current responses shown in $\boldsymbol{A}-\boldsymbol{C}$ were obtained. $\boldsymbol{E}$, The normalized TASK3 currents recorded before and during the application of 8-Br-cGMP at pH 7.4 (blue and red columns, respectively) and those recorded before and during the application of 8-Br-cGMP at $\mathrm{pH} 8.4$ (green and orange columns, respectively; $n=8$ ). All of the TASK3 currents are normalized by that obtained before the application of 8-Br-cGMP at pH 8.4. \#p < 0.05, two-way ANOVA followed by Fisher's PLSD. F, The normalized TASK1 currents recorded before and during the application of 8-Br-cGMP at pH 7.4 (blue and red columns, respectively) and those recorded before and during the application of 8-Br-cGMP at pH 8.4 (green and orange columns, respectively; $n=5$ ). All of the TASK1 currents are normalized by that obtained before the application of $8-\mathrm{Br}-\mathrm{cGMP}$ at $\mathrm{pH} 8.4$. \#p $<$ 0.05, two-way ANOVA followed by Fisher's PLSD.

sion level of TASK1 channels. The real-time RT-PCR revealed that the expression level of TASK1 channels in one large $\mathrm{MN}$ was 2.1 times higher than that in one small MN (Fig. 1F), mediating lower and higher IRs in larger and smaller MNs, respectively. Because the mean diameters of dissected small and large MNs are 15-20 and $>35 \mu \mathrm{m}$, respectively, the mean surface area in one large $M N$ is approximately three times larger than that in one small $\mathrm{MN}$. Then, the mean density of TASK1 channels in the soma of one small MN is estimated to be $\sim 1.4$ times larger than that in one large MN, suggesting that the density of TASK1 channels increases with a decrease in cell diameter, in contrast to the general assumption that the density of leak $\mathrm{K}^{+}$channels is invariable irrespective of cell sizes.
In the present study, TASK3 mRNA was hardly detected in small MNs, while TASK3 mRNA was detected half as much as TASK1 mRNA in one large MN (Fig. 1F). However, the expression level of TASK3 mRNA in large MNs may have been overestimated compared with that in small MNs because thinner dendrites expressing TASK3 in smaller MNs had been cut by LMD, whereas thicker proximal dendrites expressing TASK3 in larger MNs were well preserved as a part of the soma. Therefore, the difference in the relative value of TASK3 mRNA to TASK1 mRNA between small and large MNs may be smaller than the estimated value. In contrast with the present findings, previous studies (Karschin et al., 2001; Talley et al., 2001) using in situ hybridization demonstrated that the expression level of TASK3 mRNA in MNs appeared to be higher 
A

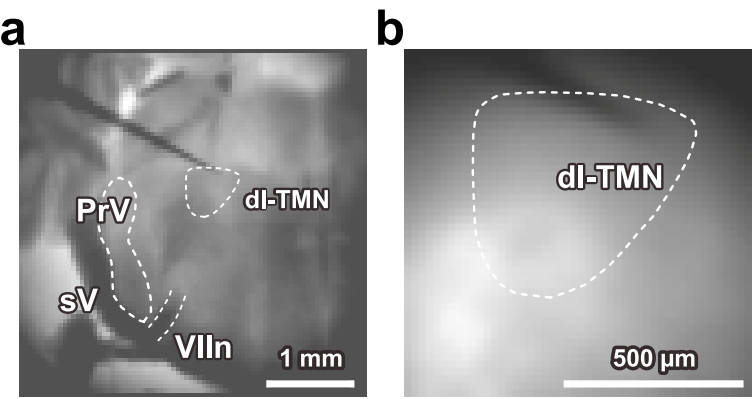

B

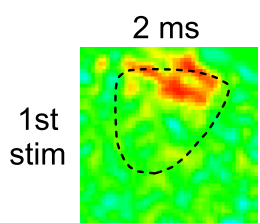

\section{Control}
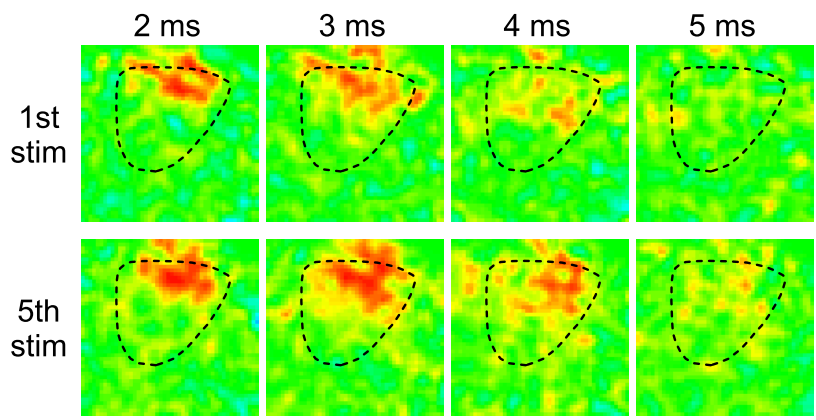

10th stim

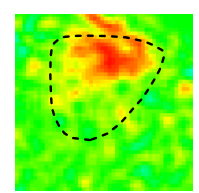

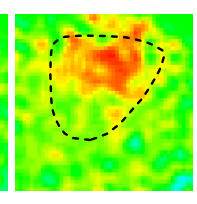

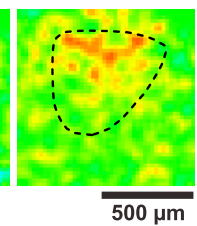

C
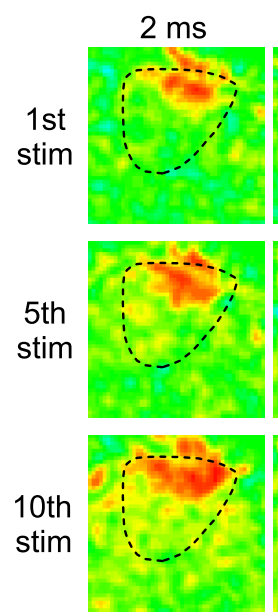

E

\section{8-Br-cGMP}
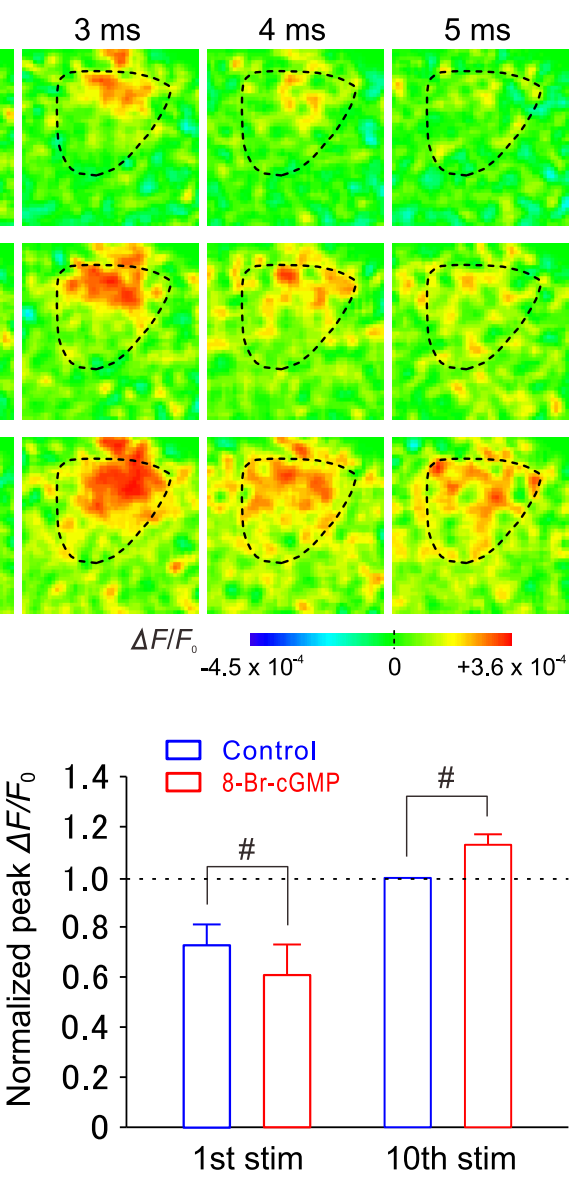

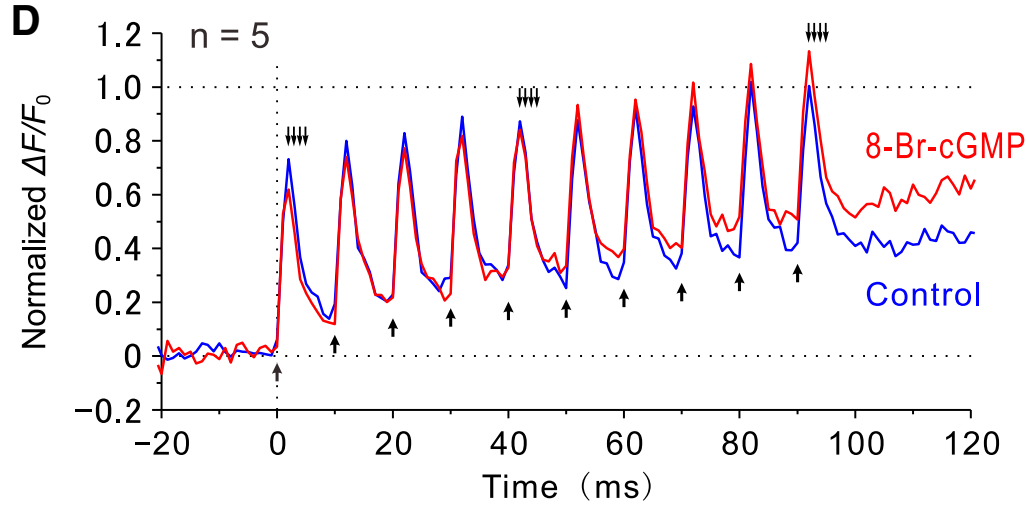

Figure 9. Recruitment of MNs in the dl-TMN caused by the stimulation of presumed la afferents. Aa, A bright-field image of a brainstem slice including the dl-TMN. $\boldsymbol{A} \boldsymbol{b}$, A resting light intensity image of the dl-TMN. $\boldsymbol{B}, \boldsymbol{C}$, Sample pseudocolor images of optical responses induced by stimulation of the dorsal part of the TMN before $(\boldsymbol{B})$ and after $(\boldsymbol{C})$ the application of 8-Br-cGMP. $\boldsymbol{D}$, Traces representing averaged temporal profiles obtained before and after the application of 8-Br-cGMP $(n=5)$. Upward arrows indicate the time points at which the stimuli were applied. Downward arrows indicate the time points at which the respective pseudocolor images were obtained $(\boldsymbol{B}, \boldsymbol{C})$. $\boldsymbol{E}$, The normalized peak amplitudes of optical responses induced by the 1st and 10th stimuli before and after the application of 8-Br-cGMP. \#p < 0.02, two-way ANOVA followed by Fisher's PLSD.

than that of TASK1 mRNA. This discrepancy can be explained by the underestimation of TASK3 mRNA levels in the present study because dendrites expressing TASK3 had been cut by LMD (Fig. 1). The group la synapses are made mostly on the dendrites of $\alpha \mathrm{MNs}$ in the dl-TMN (Dessem and Taylor, 1989; Yabuta et al., 1996). As TASK3 channels were predominantly expressed in the dendrites of MNs (Fig. 2E), dendritic la-EPSPs would be modulated largely by the activity of TASK3 channels (Fig. $6 D, E$ ). Thus, our results suggest that IRs in small $\alpha \mathrm{MNs}$ can be modulated largely by the activity of somatic TASK1 channels, while IRs in large $\alpha \mathrm{MNs}$ can be modulated largely by the activity of TASK1 and TASK3 channels. The impact of synaptic inputs can be modulated by the activity of den- 
dritic TASK3 channels in $\alpha \mathrm{MNs}$ in the dl-TMN, which may be more prominent in large $\alpha \mathrm{MNs}$ compared with small $\alpha \mathrm{MNs}$.

\section{The population of small MNs innervating JC muscles does not necessarily represent $\gamma \mathrm{MNs}$}

It has been reported (Rokx et al., 1985, 1987) that soma diameters of rat MNs innervating JC muscles displayed a bimodal distribution showing two peaks at 15 and $24 \mu \mathrm{m}$, with the boundary at $20 \mu \mathrm{m}$, and the populations of small and large MNs were considered to be $\gamma \mathrm{MNs}$ and $\alpha \mathrm{MNs}$, respectively. This is because the MNs included in the ventromedial TMN, which innervates jaw-opening muscles, displayed a unimodal distribution due to the absence of $\gamma \mathrm{MNs}$ (Rokx et al., 1985, 1987). In the present study, we aimed to obtain whole-cell recordings from large and small MNs that have polygonal or stellate-shaped somata with many dendrites (Fig. 3), but not those showing piriform or round-shaped somata with fewer primary dendrites, which are the characteristics of $\gamma \mathrm{MNs}$ (Card et al., 1986; Bae et al., 2002). Simultaneous recordings from small and large MNs revealed that la-like EPSPs were evoked in both small and large MNs simultaneously, and the small and large MNs were recruited in the sizedependent manner. These features of MNs are distinct from morphological and electrophysiological features of $\gamma$ MNs (Eccles et al., 1960; Moschovakis et al., 1991). Thus, both the small and large MNs in which whole-cell recordings were made are most likely to be $\alpha \mathrm{MNs}$.

On the other hand, we cannot rule out the possibility that in the real-time PCR study we sampled $\gamma \mathrm{MNs}$ as small MNs $(15-20 \mu \mathrm{m})$. The expression level of TASK1 channels in one large $\alpha \mathrm{MN}$ is 2.1 times higher than that in one small $\alpha \mathrm{MN}$, provided that there was no difference in the expression level of TASK1 mRNA between $\gamma \mathrm{MNs}$ and small $\alpha$ MNs (Fig. 1F). However, provided that the expression level of TASK1 channels is higher in $\gamma \mathrm{MNs}$ than in small $\alpha \mathrm{MNs}$, the expression level of TASK1 mRNA in small $\alpha \mathrm{MNs}$ may have been overestimated. On the contrary, provided that the expression level of TASK1 channels is lower in $\gamma \mathrm{MNs}$ than in $\alpha \mathrm{MNs}$, the expression level of TASK1 mRNA in small $\alpha$ MNs may have been underestimated. Thus, the value of 2.1 times might be changed depending on the expression level of TASK1 mRNA in $\gamma \mathrm{MNs}$, although this value may not be $<1$, provided that there are at least as many $\alpha \mathrm{MNs}$ as $\gamma \mathrm{MNs}$ in the population of small MNs. Indeed, the population of small MNs was found to contain as many $\alpha \mathrm{MNs}$ as $\gamma \mathrm{MNs}$, which were identified immunohistochemically using NeuN, Err3, and ChAT (Saito et al., 2013).

\section{Critical involvement of TASK channels in rank- ordered recruitment of $\alpha$ MNs in the dl-TMN}

During the slow-closing phase of the mastication cycle (Lund, 1991), the isometric contraction of JC muscles is developed through the rank-ordered recruitment of $\alpha \mathrm{MNs}$ innervating JC muscles (Yemm, 1977). In the present study, we demonstrated that the size of MNs is proportional to the expression level of somatic TASK1 channels (Fig. 1), and the IR is consequently larger in small $\alpha \mathrm{MNs}$ than in large $\alpha \mathrm{MNs}$ (Fig. 3). Furthermore, dual whole-cell recordings obtained from a pair of $\alpha \mathrm{MNs}$ of different sizes revealed that the spike threshold in the smaller $\alpha \mathrm{MN}$ is lower than that in the larger $\alpha \mathrm{MN}$ (Fig. 4) and that $\alpha \mathrm{MNs}$ are invariably recruited from the smaller to larger $\alpha \mathrm{MNs}$ in response to repetitive stimulation of the presumed la afferents (Figs. 5, 7). These findings suggest that at least somatic TASK1 channels are critically involved in the rank-ordered recruitment of $\alpha \mathrm{MNs}$ in the dl-TMN. This possibility was further confirmed by the modulation of TASK channels by 8 -Br-cGMP.

In contrast with the enhancing effects of 8-Br-cGMP on cloned TASK1 currents (Toyoda et al., 2010), cloned TASK3 channels were found to be inhibited by $8-\mathrm{Br}$ cGMP (Fig. 8). Thus, TASK1 and TASK3 channels are inversely regulated by the cGMP-PKG signaling pathway. It has been reported that NO/cGMP signaling inhibited TASK-like currents in hypoglossal MNs (Wenker et al., 2012). Because it was demonstrated that $34 \%$ and $52 \%$ of leak $\mathrm{K}^{+}$currents in hypoglossal MNs are mediated by the homodimeric TASK3 and heterodimeric TASK1/3 channels, respectively (Berg et al., 2004), the inhibitory effects of TASK-like currents by NO-cGMP-PKG pathway may have been brought about at least partly by the inhibition of homodimeric TASK3 channels, although it remains unknown how the NO-cGMP-PKG pathway modulates heterodimeric TASK $1 / 3$ channels. While it is well established that a rank-ordered recruitment of motor units occurs when increasing the bite force during voluntary isometric contraction of JC muscles (Yemm, 1977), to the best of our knowledge, there is no report showing that rank-ordered recruitment is involved in the control of tongue movement, probably because any isometric contraction does not take place in tongue muscles that have free ends. This functional difference may be reflected in the differential expression patterns of TASK channels between the $\alpha \mathrm{MNs}$ in the dl-TMN and hypoglossal MNs. If this is the case, TASK1 channels may play an important role in regulating the isometric contraction through rankordered recruitment of $\alpha \mathrm{MNs}$ compared with TASK3 or TASK $1 / 3$ channels.

Previous studies proposed that the specific membrane resistance, but not the cell size itself, is the key determinant of the orderly recruitment of spinal $\alpha \mathrm{MNs}$ (Gustafsson and Pinter, 1984a,b). This hypothesis is strongly supported by the present findings that TASK channels are differentially expressed in the small and large MNs (Fig. 1), because the density of TASK channels directly impacts membrane resistance. The expression levels of TASK channels rather than cell size itself appear to play a more fundamental role in the orderly recruitment of MNs in the dl-TMN, although the expression level of TASK1 is proportional to the size in MNs of the dl-TMN.

The orderly recruitment of MNs is determined not only by the intrinsic properties of MNs (Gustafsson and Pinter, 1985) but also by the synaptic inputs they receive (Heckman and Binder, 1988, 1993). Indeed, it has been demonstrated that not only la-EPSPs but also effective la-EPSCs were larger in higher IR MNs than in lower IR $\mathrm{MNs}$, and the la synaptic input was estimated to produce a twofold expansion of the differences in $\mathrm{MN}$ recruitment 
A Rank-order recruitment of MNs in the dI-TMN

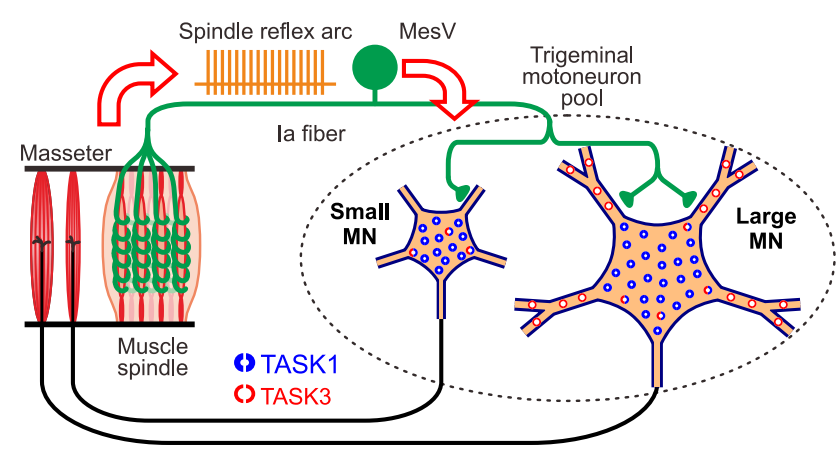

B NO inputs decreases the recruitment time

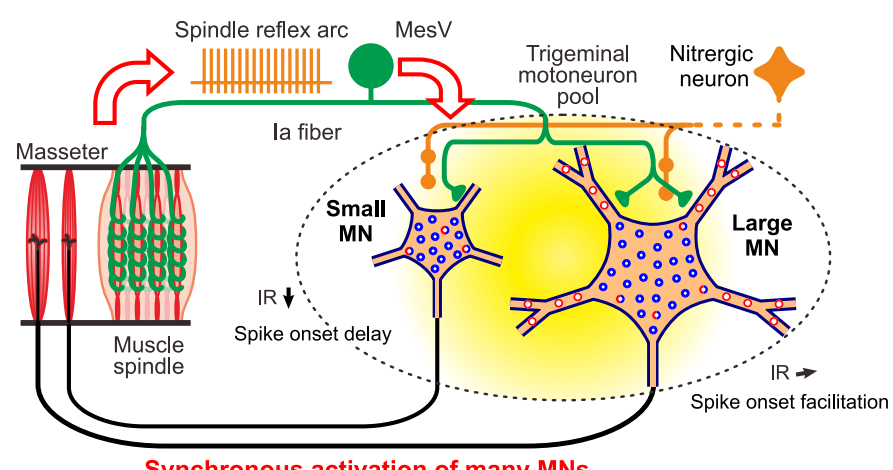

Synchronous activation of many MNs

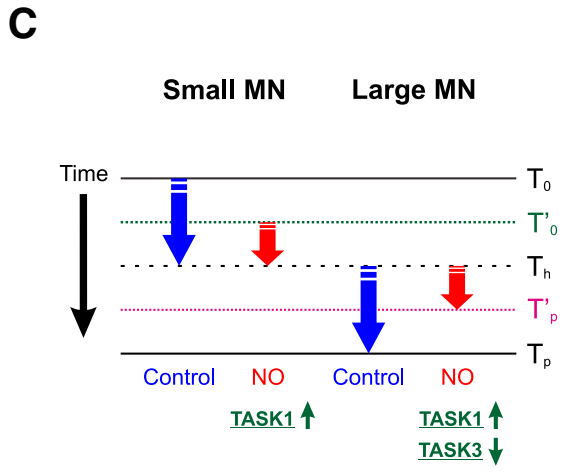

D

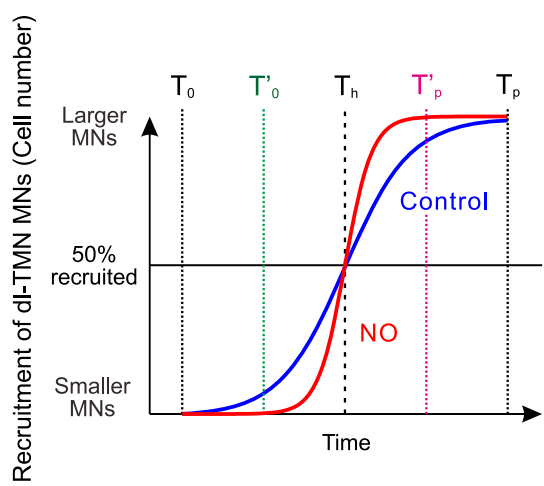

Figure 10. Rank-ordered recruitment of $\alpha \mathrm{MNs}$ in the dl-TMN and its modulation by NO inputs. $\boldsymbol{A}$, During the isometric contraction of $\mathrm{JC}$ muscles, $\alpha \mathrm{MNs}$ in the dl-TMN are recruited in rank order from smaller to larger $\alpha \mathrm{MNs}$ in response to the activation of la afferents. $\boldsymbol{B}$, When NO is released in the TMN by the activity of nitrergic neurons, IRs in small $\alpha \mathrm{MNs}$ are decreased, and subsequently increase the spike-onset latencies. By contrast, in relatively large $\alpha \mathrm{MNs}$, NO either hardly changes or slightly increases IRs, subsequently causing no increase or a slight decrease in the spike-onset latencies, while it facilitates recruitment of the largest $\alpha \mathrm{MNs}$. Consequently, NO causes a more synchronous activation of smaller and larger $\alpha \mathrm{MNs}$. C. Alteration of the recruitment of small and large $\alpha \mathrm{MNs}$ in the dl-TMN by the activation of NO inputs. $\mathrm{T}_{0}$, Recruitment-onset time; $\mathrm{T}_{\mathrm{h}}$, time at which half of $\alpha \mathrm{MNs}$ are recruited and from which large $\alpha \mathrm{MNs}$ start to be recruited; $\mathrm{T}_{\mathrm{p}}$, time at which all the $\alpha \mathrm{MNs}$ are recruited; $\mathrm{T}_{0}{ }_{0}$, recruitment onset time after the activation of NO inputs; T', time at which all the $\alpha \mathrm{MNs}$ are recruited after the activation of NO inputs. $\boldsymbol{D}$, Schematic diagram of the slow and fast rank-ordered recruitment observed before and after the activation of NO inputs, respectively. Slow precise and fast ballistic force increases can be achieved through the modulation of TASK1/TASK3 channels by NO inputs.

thresholds that are generated by intrinsic cellular properties (Heckman and Binder, 1988). This finding is consistent with previous studies showing that the number of la synaptic terminals was larger (Burke, 1968) and that their release probabilities were higher (Honig et al., 1983; Collins et al., 1984) in MNs with higher IR than in MNs with lower IR, although these differences were not large ( $\mathrm{Pe}$ shori et al., 1998). If this is the case, the larger effective la-EPSCs in higher IR MNs may serve to increase the safety factor to ensure the orderly recruitment of MNs even under the condition that TASK1 and TASK3 channels are fully activated and suppressed, respectively, by 8-Br-cGMP.

\section{8-Br-cGMP modulates the rank-ordered recruitment of $\alpha \mathrm{MNs}$ through differential modulation of small and large $\alpha \mathrm{MNs}$}

In small $\alpha \mathrm{MNs}$, 8-Br-cGMP hyperpolarized the resting membrane potentials and decreased the IRs (Figs. $6 A$,
$7 A$ ), and subsequently increased the latencies to the first spikes (Fig. 7A,C). As TASK1 channels are expressed in the somata of MNs (Figs. 1, 2), these results could be due to the enhancement of TASK1 currents by activation of the NO-cGMP-PKG pathway. In contrast, in large $\alpha \mathrm{MNs}$, 8-Br-cGMP hardly or slightly depolarized the resting membrane potentials and hardly or slightly increased the IRs (Figs. 6B, 7B), subsequently causing no increase or a slight decrease in the spike-onset latencies (Fig. 7B,C). Because TASK1 and TASK3 channels are inversely regulated by 8-Br-cGMP (Fig. 8), the decreases in IRs through the activation of somatic TASK1 channels by $8-\mathrm{Br}-\mathrm{cGMP}$ may be canceled by increases in IRs through the inhibition of dendritic TASK3 channels by 8-Br-cGMP. However, the modest effect of 8 -Br-cGMP on TASK3 compared with TASK1 channels appeared to be contradictory to the negligible effect of 8-Br-cGMP on the IRs in large $\alpha$ MNs. Nevertheless, this was not the case. TASK3 currents recorded in Xenopus oocytes were 5 to 10 times larger 
compared with TASK1 currents despite the fact that the number of cRNAs for TASK1 injected into Xenopus oocytes was the same as that for TASK3 (see Materials and Methods). This suggests that the expression level of TASK3 channels might have been much higher than that of TASK1 channels in Xenopus oocytes. Provided that PKG activation by 8-Br-cGMP is not enough to phosphorylate all the TASK3 channel substrates in single Xenopus oocytes, the effects of 8-Br-cGMP on TASK3 currents would be small. Thus, it is not reasonable to directly compare the effects of 8 -Br-cGMP on TASK1 currents with that on TASK3 currents in Xenopus oocytes. Therefore, the modest effect of 8-Br-cGMP on TASK3 channels compared with TASK1 channels is not necessarily contradictory to the negligible effect of 8-Br-cGMP on the IRs in large $\alpha \mathrm{MNs}$. These results suggest that $8-\mathrm{Br}$-cGMP decreases the time necessary for recruitment starting with smaller $\alpha \mathrm{MNs}$ and progressing to larger $\alpha \mathrm{MNs}$ (Fig. 7C). Consistently, the voltage-sensitive dye imaging revealed that 8 -Br-cGMP suppressed early optical responses but facilitated late optical responses to 10 repetitive stimulations applied at $100 \mathrm{~Hz}$, indicating inhibition of the recruitment of smaller $\alpha \mathrm{MNs}$ and the facilitation of the recruitment of larger $\alpha \mathrm{MNs}$, respectively (Fig. 9).

Together, given NO release in the TMN from nitrergic neurons during the isometric contraction of $\mathrm{JC}$ muscles (Fig. 10A), the spike-onset latency would increase in small $\alpha \mathrm{MNs}$, but would slightly decrease or remain unchanged in large $\alpha \mathrm{MNs}$, consequently decreasing the time necessary for the entire recruitment of $\alpha \mathrm{MNs}$ to lead to a synchronous activation of many $\alpha \mathrm{MNs}$ in the dl-TMN (Fig. $10 B-D)$. To execute a ballistic movement or muscle contraction, many $\alpha \mathrm{MNs}$ must be activated almost synchronously. Thus, slow and precise or fast and ballistic clenching may be achieved by the modulation of rankordered recruitment through the modulation of TASK1/ TASK3 channels presumably by NO inputs. Based on the findings that 8-Br-cGMP almost exclusively affected the leak $\mathrm{K}^{+}$currents without marked effects on the $I_{\text {ir }}$ (Fig. $6 A c)$, it is strongly suggested that TASK channels play crucial roles in the rank-ordered recruitment of MNs in the dl-TMN. However, it should be noted that the linkage between TASK channels and the orderly recruitment of MNs derived from the findings obtained by using 8-BrcGMP may be less direct compared with a demonstration using TASK channel knock-out mice.

\section{References}

Abudara V, Alvarez AF, Chase MH, Morales FR (2002) Nitric oxide as an anterograde neurotransmitter in the trigeminal motor pool. J Neurophysiol 88:497-506.

Bae YC, Choi BJ, Lee MG, Lee HJ, Park KP, Zhang LF, Honma S, Fukami H, Yoshida A, Ottersen OP, Shigenaga Y (2002) Quantitative ultrastructural analysis of glycine- and gamma-aminobutyric acid-immunoreactive terminals on trigeminal alpha- and gammamotoneuron somata in the rat. J Comp Neurol 442:308-319. CrossRef

Bawa P, Binder MD, Ruenzel P, Henneman E (1984) Recruitment order of motoneurons in stretch reflexes is highly correlated with their axonal conduction velocity. J Neurophysiol 52:410-420. Medline
Bayliss DA, Sirois JE, Talley EM (2003) The TASK family: two-pore domain background $\mathrm{K}^{+}$channels. Mol Interv 3:205-219. CrossRef Medline

Berg AP, Talley EM, Manger JP, Bayliss DA (2004) Motoneurons express heteromeric TWIK-related acid-sensitive $\mathrm{K}^{+}$(TASK) channels containing TASK-1 (KCNK3) and TASK-3 (KCNK9) subunits. J Neurosci 24:6693-6702. CrossRef Medline

Bredt DS, Glatt CE, Hwang PM, Fotuhi M, Dawson TM, Snyder SH (1991) Nitric oxide synthase protein and mRNA are discretely localized in neuronal populations of the mammalian CNS together with NADPH diaphorase. Neuron 7:615-624. Medline

Burke RE (1968) Group la synaptic input to fast and slow twitch motor units of cat triceps surae. J Physiol 196:605-630. Medline

Burke RE (1975) Motor unit properties and selective involvement in movement. Exerc Sport Sci Rev 3:31-81. Medline

Card JP, Riley JN, Moore RY (1986) The motor trigeminal nucleus of the rat: analysis of neuronal structure and the synaptic organization of noradrenergic afferents. J Comp Neurol 250:469-484. CrossRef Medline

Collins WF 3rd, Honig MG, Mendell LM (1984) Heterogeneity of group la synapses on homonymous alpha-motoneurons as revealed by high-frequency stimulation of la afferent fibers. J Neurophysiol 52:980-993.

Curtis DR, Eccles JC (1960) Synaptic action during and after repetitive stimulation. J Physiol 150:374-398. Medline

Czirják G, Enyedi P (2002) Formation of functional heterodimers between the TASK-1 and TASK-3 two-pore domain potassium channel subunits. J Biol Chem 277:5426-5432. CrossRef Medline

Dascal N (1987) The use of Xenopus oocytes for the study of ion channels. CRC Crit Rev Biochem 22:317-387. Medline

Dessem D, Taylor A (1989) Morphology of jaw-muscle spindle afferents in the rat. J Comp Neurol 282:389-403. CrossRef

Eccles JC, Eccles RM, Iggo A, Lundberg A (1960) Electrophysiological studies on gamma motoneurones. Acta Physiol Scand 50:3240. CrossRef Medline

Enyedi P, Czirják G (2010) Molecular background of leak K ${ }^{+}$currents: two-pore domain potassium channels. Physiol Rev 90:559-605. CrossRef Medline

Grimwood P, Appenteng K (1995) Effects of afferent firing frequency on the amplitude of the monosynaptic EPSP elicited by trigeminal spindle afferents on trigeminal motoneurones. Brain Res 689:299303. Medline

Grinvald A, Anglister L, Freeman JA, Hildesheim R, Manker A (1984) Real-time optical imaging of naturally evoked electrical activity in intact frog brain. Nature 308:848-850. Medline

Gustafsson B, Pinter MJ (1984a) Relations among passive electrical properties of lumbar alpha-motoneurones of the cat. J Physiol 356:401-431.

Gustafsson B, Pinter MJ (1984b) An investigation of threshold properties among cat spinal alpha-motoneurones. J Physiol 357:453483. Medline [Mismatch]

Gustafsson B, Pinter MJ (1985) On factors determining orderly recruitment of motor units: a role for intrinsic membrane properties. Trends Neurosci 8:431-433. CrossRef

Heckman CJ, Binder MD (1988) Analysis of effective synaptic currents generated by homonymous la afferent fibers in motoneurons of the cat. J Neurophysiol 60:1946-1966.

Heckman CJ, Binder MD (1993) Computer simulations of the effects of different synaptic input systems on motor unit recruitment. $J$ Neurophysiol 70:1827-1840. Medline

Henneman E (1991) The size principle and its relation to transmission failure in la projections to spinal motoneurons. Ann N Y Acad Sci 627:165-168. Medline

Henneman E, Somjen G, Carpenter DO (1965) Functional significance of cell size in spinal motoneurons. J Neurophysiol 28:560580. Medline

Honig MG, Collins WF 3rd, Mendell LM (1983) Alpha-motoneuron EPSPs exhibit different frequency sensitivities to single la-afferent fiber stimulation. J Neurophysiol 49:886-901. 
Inanobe A, Fujita S, Makino Y, Matsushita K, Ishii M, Chachin M, Kurachi $Y$ (2001) Interaction between the RGS domain of RGS4 with $G$ protein alpha subunits mediates the voltage-dependent relaxation of the $G$ protein-gated potassium channel. J Physiol 535:133-143. CrossRef

Kang D, Han J, Talley EM, Bayliss DA, Kim D (2004) Functional expression of TASK-1/TASK-3 heteromers in cerebellar granule cells. J Physiol 554:64-77. CrossRef Medline

Kang Y, Saito M, Sato H, Toyoda H, Maeda Y, Hirai T, Bae YC (2007a) Involvement of persistent $\mathrm{Na}+$ current in spike initiation in primary sensory neurons of the rat mesencephalic trigeminal nucleus. J Neurophysiol 97:2385-2393.

Kang Y, Dempo Y, Ohashi A, Saito M, Toyoda H, Sato H, Koshino H, Maeda Y, Hirai T (2007b) Nitric oxide activates leak K+ currents in the presumed cholinergic neuron of basal forebrain. J Neurophysiol 98:3397-3410.

Karschin C, Wischmeyer E, Preisig-Müller R, Rajan S, Derst C, Grzeschik KH, Daut J, Karschin A (2001) Expression pattern in brain of TASK-1, TASK-3, and a tandem pore domain $\mathrm{K}^{+}$channel subunit, TASK -5 , associated with the central auditory nervous system. Mol Cell Neurosci 18:632-648. CrossRef

Lauritzen I, Zanzouri M, Honoré E, Duprat F, Ehrengruber MU, Lazdunski M, Patel AJ (2003) $\mathrm{K}^{+}$-dependent cerebellar granule neuron apoptosis. Role of task leak $\mathrm{K}^{+}$channels. J Biol Chem 278:3206832076. CrossRef Medline

Leonoudakis D, Gray AT, Winegar BD, Kindler CH, Harada M, Taylor DM, Chavez RA, Forsayeth JR, Yost CS (1998) An open rectifier potassium channel with two pore domains in tandem cloned from rat cerebellum. J Neurosci 18:868-877. Medline

Lesage $F$ (2003) Pharmacology of neuronal background potassium channels. Neuropharmacology 44:1-7. Medline

Limwongse V, DeSantis M (1977) Cell body locations and axonal pathways of neurons innervating muscles of mastication in the rat. Am J Anat 149:477-488. CrossRef Medline

Lund JP (1991) Mastication and its control by the brain stem. Crit Rev Oral Biol Med 2:33-64. Medline

Mizuno N, Konishi A, Sato M (1975) Localization of masticatory motoneurons in the cat and rat by means of retrograde axonal transport of horseradish peroxidase. J Comp Neurol 164:105-115. CrossRef Medline

Moschovakis AK, Burke RE, Fyffe RE (1991) The size and dendritic structure of HRP-labeled gamma motoneurons in the cat spinal cord. J Comp Neurol 311:531-545. CrossRef Medline

Peshori KR, Collins WF, Mendell LM (1998) EPSP amplitude modulation at the rat la-alpha motoneuron synapse: effects of $G A B A_{B}$ receptor agonists and antagonists. J Neurophysiol 79:181-189. Medline

Pfaffl MW (2001) A new mathematical model for relative quantification in real-time RT-PCR. Nucleic Acids Res 29:e45. Medline

Pose I, Fung S, Sampogna S, Chase MH, Morales FR (2005) Nitrergic innervation of trigeminal and hypoglossal motoneurons in the cat. Brain Res 1041:29-37. CrossRef Medline
Rokx JT, van Willigen JD, Jüch PJ (1985) Distribution of innervating neurons of masticatory muscle spindles in the rat: an HRP study. Exp Neurol 88:562-569. Medline

Rokx JT, Liem RS, van Willigen JD (1987) Identification of alpha and gamma trigeminal motoneurons by the vibratome paraplast technique for HRP histochemistry. Acta Anat (Basel) 129:333-336. Medline

Saito M, Isogai-Morita Y, Emura N, Toyoda H, Sato H, Kang Y (2013) Rank-ordered recruitment of jaw-closing alpha-motoneurons depending on the activation of TASK1 and TASK3 channels in the rat. Soc Neurosci Abstr 43:320.17

Sato $H$, Shimanuki $Y$, Saito $M$, Toyoda H, Nokubi T, Maeda $Y$, Yamamoto T, Kang Y (2008) Differential columnar processing in local circuits of barrel and insular cortices. J Neurosci 28:30763089. CrossRef Medline

Shigenaga Y, Mitsuhiro Y, Yoshida A, Cao CQ, Tsuru H (1988) Morphology of single mesencephalic trigeminal neurons innervating masseter muscle of the cat. Brain Res 445:392-399. Medline

Shigenaga Y, Mitsuhiro Y, Shirana Y, Tsuru H (1990) Two types of jaw-muscle spindle afferents in the cat as demonstrated by intraaxonal staining with HRP. Brain Res 514:219-237. Medline

Talley EM, Solorzano G, Lei Q, Kim D, Bayliss DA (2001) CNS distribution of members of the two-pore-domain (KCNK) potassium channel family. J Neurosci 21:7491-7505. Medline

Toyoda H, Saito M, Sato H, Dempo Y, Ohashi A, Hirai T, Maeda Y, Kaneko T, Kang Y (2008) cGMP activates a pH-sensitive leak $\mathrm{K}^{+}$ current in the presumed cholinergic neuron of basal forebrain. $J$ Neurophysiol 99:2126-2133. CrossRef

Toyoda H, Saito M, Okazawa M, Hirao K, Sato H, Abe H, Takada K, Funabiki K, Takada M, Kaneko T, Kang Y (2010) Protein kinase G dynamically modulates TASK1-mediated leak $\mathrm{K}^{+}$currents in cholinergic neurons of the basal forebrain. J Neurosci 30:5677-5689. CrossRef

Travers JB, Yoo JE, Chandran R, Herman K, Travers SP (2005) Neurotransmitter phenotypes of intermediate zone reticular formation projections to the motor trigeminal and hypoglossal nuclei in the rat. J Comp Neurol 488:28-47. CrossRef

Wenker IC, Benoit JP, Chen X, Liu H, Horner RL, Mulkey DK (2012) Nitric oxide activates hypoglossal motoneurons by cGMPdependent inhibition of TASK channels and cGMP-independent activation of HCN channels. J Neurophysiol 107:1489-1499. CrossRef Medline

Yabuta NH, Yasuda K, Nagase Y, Yoshida A, Fukunishi Y, Shigenaga $Y$ (1996) Light microscopic observations of the contacts made between two spindle afferent types and alpha-motoneurons in the cat trigeminal motor nucleus. J Comp Neurol 374:436-450. CrossRef Medline

Yemm R (1977) The orderly recruitment of motor units of the masseter and temporal muscles during voluntary isometric contraction in man. J Physiol 265:163-174. CrossRef 